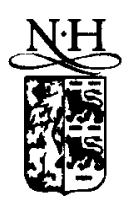

\title{
A ncw frictional time intcgration algorithm for large slip multi-body frictional contact problems
}

\author{
C. Agelet de Saracibar \\ ETS Ingenieros de Caminos, Canales y Puertos, Universitat Politècnica de Catalunya, Barcelona, Spain
}

Received 8 August 1995

\begin{abstract}
In this paper a new frictional time integration suitable for large slip multibody frictional contact problems is presented. The algorithm is introduced within the simple context of a model problem: the sliding motion of a particle onto a rough surface. Time integration of frictional traction is performed introducing a new slip path parametrization, which is defined independently of the local surface finite element parametrization used in the spatial triangularization. The key point of the algorithm is that now, in presence of large slips, problems associated with slip motions such that a full incremental slip path is not within a single surface element, are completely bypassed. Remarkably, the algorithm is defined on the sole basis of the unit outward normal field to the surface without any appeal to the underlying local surface finite element triangularization. Geometrically, the assumed slip path can be viewed as an approximation to the geodesic passing throughout the initial and final points of each incremental slip path. The algorithm is amenable to exact linearization and asymptotic quadratic rate of convergence can be achieved within a Newton-Raphson iterative solution scheme. The algorithm can easily be extended to large slip multi-body frictional contact problems, involving finite strains.
\end{abstract}

\section{Introduction. Motivation and goals}

Mathematically, the numerical analysis of frictional contact problems amounts to find the solution of an Initial Boundary Value Problem (IBVP) within a constrained solution space. Then a weak formulation of the IBVP leads to variational inequalities. Significant references on variational inequalities are the books of Duvaut and Lions [1] and Kikuchi and Oden [2]. A regularization of the frictional contact constraints, using penalty or augmented Lagrangian methods, allows to bypass the need to find a solution within a constrained solution space and provides a very convenient displacementdriven frictional contact formulation. The penalty method can be considered as the standard regularization procedure and it has been used by Oden and Pires [3], Cheng and Kikuchi [4], Hallquist et al. [5], Simo et al. [6], Curnier and Alart [7], Wriggers et al. [8], Belytschko and Neal [9], Laursen [10], Laursen and Simo [11,12] and Agelet de Saracibar $[13,14]$ among others. To avoid well-known problems inherent to the penalty method, such as penalty sensitivity and possible ill-conditioning of the system of equations, while retaining his advantages, the augmented Lagrangian method has been used as an alternative regularization procedure. Within the frictional contact problems context, the

\footnotetext{
"This paper is dedicated to the memory of Prof. Juan C. Simo. Since February 1993 to February 1994 I had the privilege and the unique experience to work with him, while I was at the Division of Applied Mechanics of the Stanford University, for a sabbatical stage under his advice. His enthusiastic support, motivation, expertise and the large moments of fruitful discussion, developing algorithms at the campus cafeteria, will never be forgotten. This paper, in which he was involved, is a result of some of these hours we expend together.
} 
augmented Lagrangian method has been used by Laursen [10], Simo and Laursen [15], Laursen and Simo $[11,16]$ and Laursen and Govindjee [17].

A fully nonlinear kinematics formulation of frictionless contact problems, including the derivation of the algorithmic contact operators, was developed by Wriggers and Simo [18] for 2D linear surface elements and by Parish [19] for 3D linear surface elements. An extension to frictional contact problems for 2D linear surface elements was provided by Wriggers [20]. A general fully nonlinear kinematics formulation of multi-body frictional contact problems at finite strain, fully developed on a continuum setting for 2D and 3D problems, was first given by Laursen and Simo [12]. A further extension to incorporate a frictional wear model was given by Agelet de Saracibar [13].

Furthermore, a displacement-driven formulation of frictional contact problems, allows to widely exploit the framework developed for computational plasticity. See Simo and Hughes [21] and Simo [22] for an excellent presentation of current topics and last developments in computational plasticity. In particular, return mapping algorithms originally developed for plasticity can be applied to integrate the frictional traction. The lowest Backward-Difference (BD) method, the Backward-Euler (BE) method, has become in the last years, the standard algorithm for the frictional time integration of the regularized frictional constrained evolution problem. Frictional return mapping, using the $\mathrm{BE}$ algorithm, have been used by Wriggers [20], Giannakopoulos [23], Wriggers et al. [8], Laursen and Simo [12,16] and Agelet de Saracibar [13,14], among others. Recently, an Implicit Runge-Kutta (IRK) method, the generalized Projected Mid-Point (PMP) algorithm was proposed, within the context of J2 plasticity, by Simo [22]. A frictional return mapping using the generalized PMP algorithm has been proposed by Agelet de Saracibar $[13,14]$.

In the general fully nonlinear formulation of multi-body frictional contact problems, fully developed

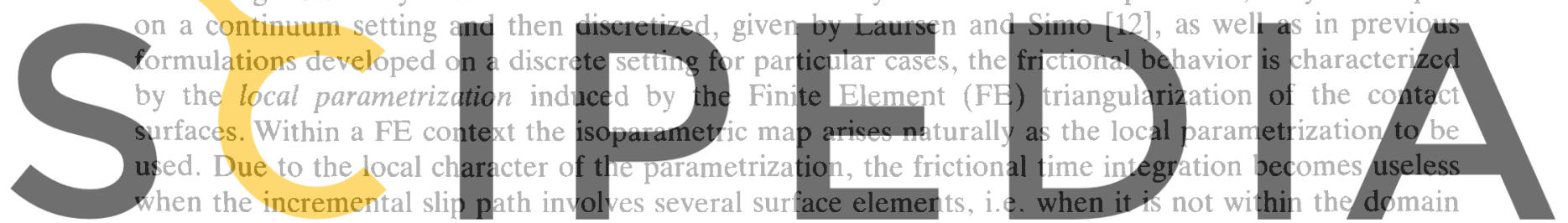

of the local parameterization of a single surface element of the finite element triangularization.

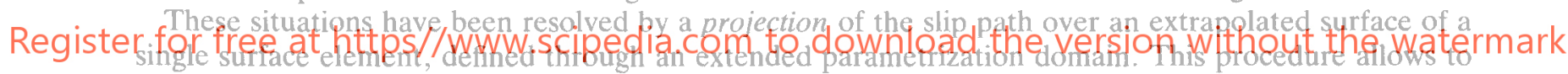

use a single local parametrization (within an extended domain) where a slip path belongs to different

surface elements, each one described with a local parametrization. Such procedures, although usually not addressed in the literature, can be considered as a standard practice in several computer codes as DYNA or FEAP, for example.

Some of the drawbacks of these projection procedures are the following:

(i) The projected slip path amounts to find a projection lying outside the limits of the local parametrization domain. Note that, the orientation preserving map induced by the local parametrization, is guaranteed to exist only within the underlying domain. Then, situations may arise in which the projection points are located in areas with negative or zero jacobian, leading to a useless algorithm.

(ii) The slip amount is one of the main parameters for the characterization of wear phenomena in multi-body frictional wear contact problems. See, for example, Agelet de Saracibar [13] for a numerical model of multi-body frictional wear contact problems. Projection of slip path can lead to an important underevaluation of the slip amount, and hence, to an underevaluation of frictional traction, frictional dissipation and wear estimate.

The above considerations have motivated the need to get a new frictional time integration algorithm suitable for large slip multi-body frictional contact problems. This new algorithm must be equipped with the following features:

(i) Slip path projection-based procedures must be avoided, due to the drawbacks mentioned above.

(ii) Usc of local surface elements parametrization must be avoided.

(iii) A new slip path parametrization suitable for large slips must be introduced.

(iv) The new frictional time integration algorithm must be amenable to an exact linearization, to preserve quadratic asymptotic rate of convergence typical of a Newton-Raphson solution scheme. 
With these considerations in mind, a new algorithm for the time integration of frictional traction is proposed. To introduce the main idea of the new algorithm it suffices to consider a simple model problem: the sliding motion of a particle onto a rough surface.

The remaining of the paper is as follows. Section 2 deals with the set up of the model problem. Holonomic and nonholonomic constraints are introduced and the regularized problem is considered. Use of a product formula algorithm and an operator split motivates the subsequent numerical integration of the constrained evolution problem. In Section 3, the new frictional time integration algorithm, suitable for large slip multi-body frictional contact problems, is introduced within the simplest context of the model problem. Section 4 deals with representative numerical simulations and some concluding remarks are given in Section 5. The extension of the algorithm for a general fully nonlinear multi-body frictional contact problem is included in Appendix A and the exact linearization of the new frictional time integration algorithm is given in Appendix B.

\section{A model problem: sliding motion of a particle onto a surface.}

The main aspects involved in the new frictional time integration algorithm of the constrained frictional evolution problem, can conveniently be introduced within the framework of a simple model problem: the dynamic motion of a particle sliding onto a rigid rough surface.

The kinematic of the sliding motion of a particle onto a rigid rough surface constitutes an example of motion of a particle subjected to holonomic and non-holonomic constraints. Kinematic holonomic constraints on the particle arises from the condition to lie onto the rigid surface. Non-holonomic

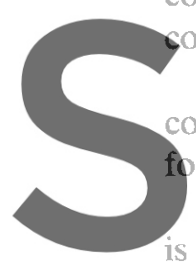
nstraints arises from the frictional conditions on the surfac The regularized problen ontext of the product for

llowing numerical integ One of the key points of to be able to compute the slip andunt within a typiser
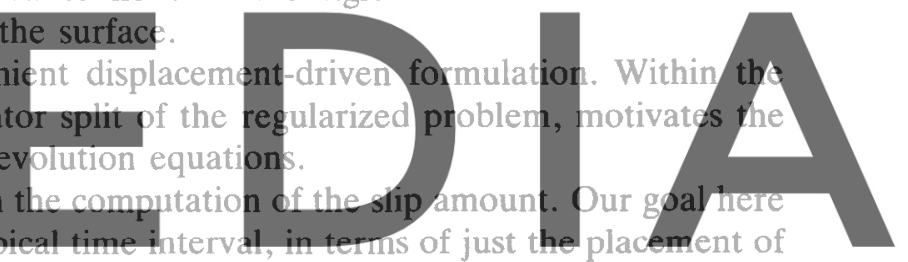

the particle and the unit outward normal to the surface at the beginning and the end of the time

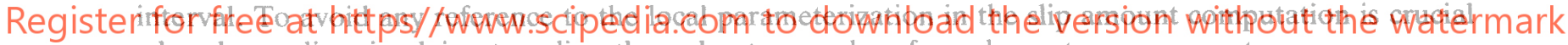
when large slips, implying traveling throughout several surface elements, are present.

\subsection{Description of the problem}

Let $2 \leqslant n_{\text {dim }} \leqslant 3$ be the space dimension and $I:=[0, T] \subset \mathbb{R}_{+}$the time interval of interest. Let $X$ be the reference placement of a particle $\mathscr{P}$ constrained to lie onto a rigid rough smooth surface $\Gamma$. Denote by $\varphi: \mathscr{P} \times I \rightarrow \mathbb{R}^{n} \mathrm{dim}$ the motion map of the particle $\mathscr{P}$, with material velocity $V:=\partial_{t} \varphi$. For each time $t \in I$, the mapping $t \in I \mapsto \varphi_{t}:=\varphi^{(i)}(\cdot, t)$ represents a one-parameter family of configurations indexed by time $t$, which maps the reference placement of the particle $\mathscr{P}$ onto its current placement. The current placement of the particle at time $t \in I$ will be denoted as $x:=\varphi_{t}(X)=\varphi(X, t)$.

Let $\Gamma$ be an oriented smooth rigid surface defined as the set

$$
\Gamma:=\left\{\boldsymbol{Y} \in \mathbb{R}^{n} \mathrm{dim}: g_{\mathrm{N}}(\boldsymbol{Y})=0\right\}
$$

with unit outward normal $\nu \in S^{2}$ defined as

$$
\nu(\boldsymbol{Y}):=-\nabla g_{\mathrm{N}}(\boldsymbol{Y}) /\left\|\nabla g_{\mathrm{N}}(\boldsymbol{Y})\right\|
$$

where $S^{2}:=\left\{\boldsymbol{\nu} \in \mathbb{R}^{n_{\mathrm{dim}}}:\|\boldsymbol{\nu}\|=1\right\}$ is the unit sphere.

The dynamics of a particle sliding onto a rigid rough surface provides an example of motion of a particle subjected to holonomic and non-holonomic constraints. Fig. 1 shows the description of the model problem to be considered.

(A) Holonomic unilateral constraints. The holonomic unilateral constraints arises from the fact that the motion of the particle is constrained by the rigid surface. 


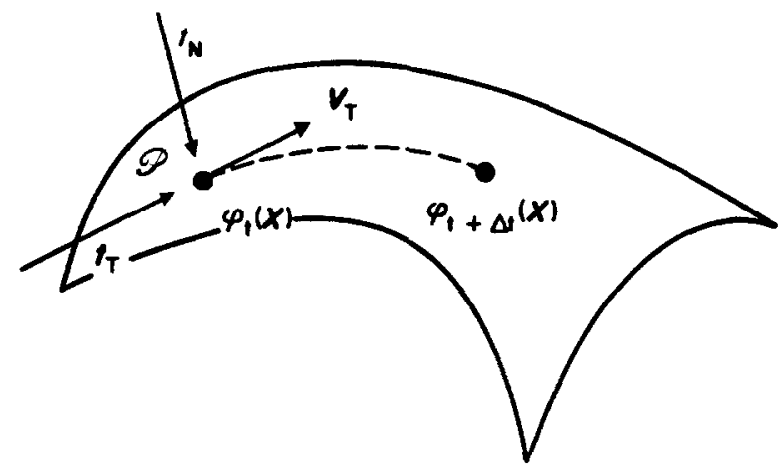

Fig. 1. Description of the model problem: Sliding motion of a particle over a rough surface.

(i) Impenetrability constraint. The presence of the rigid surface acting as an obstacle to the free motion of the particle induces an impenetrability constraint leading to an holonomic unilateral constraint on the admissible configurations of the particle. Without loss of generality, we will assume that the motion of the particle $\mathscr{P}$ with reference placement $X$ at any time $t \in I$ is constrained by the following holonomic unilateral condition

$$
g_{N} \circ \varphi(X, t) \leqslant 0
$$

(ii) Non-adhesion constraint. When the particle is on the surface, interacting tractions arises due to

he impenetrability constra

mpenetrability constraint

urface induces a friction traction on the particle,

iding motion of the particle on the surface.

Denoting by $t(\mathbb{X}, t)$ the reaction on the particle,
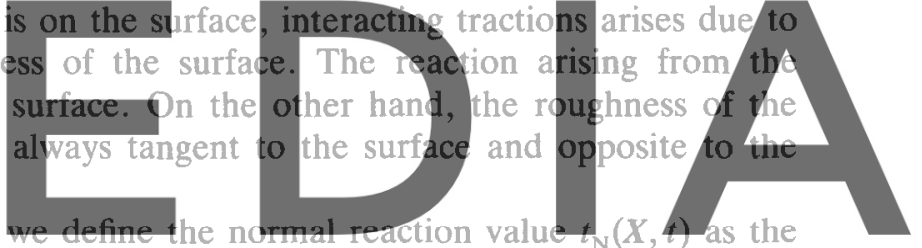

projection of $t(X, t)$ onto the unit outward normal to the surface $\boldsymbol{\nu}$, and consider the following split into

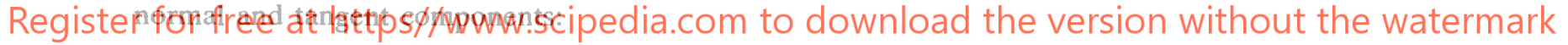

$$
t(\mathbb{X}, t):=t_{\mathrm{N}}(\mathbb{X}, t) \nu \circ \varphi(\mathbb{X}, t)+\mathbb{P}_{\nu} t(\mathbb{X}, t)
$$

With the preceding definitions the unilateral non-adhesion constraint implies that the normal reaction value must be non-negative when the particle is on the surface and zero otherwise. Mathematically, this condition takes the form:

$$
\begin{array}{ll}
t_{\mathrm{N}}(X, t) \geqslant 0 & \text { if } g_{\mathrm{N}}{ }^{\circ} \varphi(X, t)=0 \\
t_{\mathrm{N}}(X, t)=0 & \text { if } g_{\mathrm{N}}{ }^{\circ} \varphi(X, t)<0
\end{array}
$$

(iii) Contact persistency condition. This condition requires that the rate of separation of the particle of the surface must be zero for positive contact normal traction. Mathematically, this persistency condition takes the form:

$$
t_{\mathrm{N}}(X, t) \nabla g_{\mathrm{N}}{ }^{\circ} \varphi(X, t) \cdot V(X, t)=0
$$

Using the definition of the unit outward normal given by (2.2), this persistency condition can be written as

$$
t_{\mathrm{N}}(X, t) V(X, t) \cdot \nu \circ \varphi(X, t)=0
$$

and states that the normal component of the velocity of the particle must be zero for non-zero (positive) contact normal traction.

The above constraints set of impenetrability of the rigid obstacle, non-adherence to the surface and contact persistency, can be expressed in the form of Kuhn-Tucker complementarity conditions as 


$$
\begin{aligned}
& g_{\mathrm{N}} \circ \varphi(X, t) \leqslant 0 \\
& t_{\mathrm{N}}(X, t) \geqslant 0 \\
& t_{\mathrm{N}}(X, t) g_{\mathrm{N}}{ }^{\circ} \varphi(X, t)=0 \\
& t_{\mathrm{N}}(X, t) \nabla g_{\mathrm{N}}{ }^{\circ} \varphi(X, t) \cdot V(X, t)=0
\end{aligned}
$$

(B) Non-holonomic frictional constraints. The frictional behavior due to the asperities of the rough surface, induces non-holonomic constraints on the admissible configurations of the particle.

Consider the following split of the velocity $V(X, t)$ of the particle sliding onto the surface:

$$
V(X, t):=V_{\mathrm{N}}(X, t) \nu \circ \varphi(X, t)+V_{\mathrm{T}}
$$

where $V_{\mathrm{N}}(X, t)$ is the projection of the velocity onto the unit outward normal, i.e. the rate of separation of the particle, and $V_{\mathrm{T}}:=\mathbb{P}_{\nu} \boldsymbol{V}$ is the projection of the velocity onto the tangent plane to the surface.

As it was shown above, the contact persistency condition leads to the requirement that the rate of separation of the particle of the surface must be zero for positive contact normal traction. Using (2.7) and (2.9) this condition leads to the constraint

$$
V_{\mathrm{N}}=0 \quad \text { if } t_{\mathrm{N}}(\boldsymbol{X}, t)>0
$$

\section{and then}

$$
V_{\mathrm{T}}:=\mathbb{P}_{\nu} \boldsymbol{V}=\boldsymbol{V} \text { if } t_{\mathrm{N}}(\boldsymbol{X}, t)>0
$$

The one-form associated to the slip velocity will be denoted as $V_{T}^{b}$.

\section{Let $\boldsymbol{t}_{\mathrm{T}}(\boldsymbol{X}, t)$ be defined}

plane, as
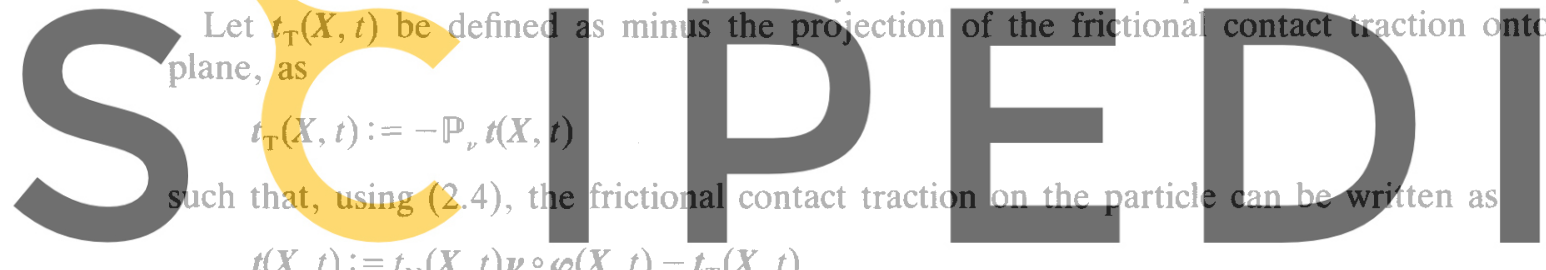

$$
t(X, t):=t_{\mathrm{N}}(X, t) \nu \circ \varphi(X, t)-t_{\mathrm{T}}(\boldsymbol{X}, t)
$$

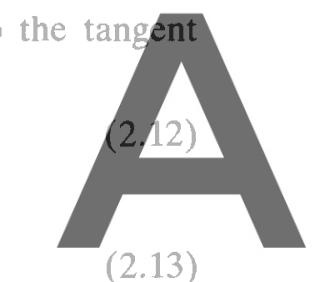

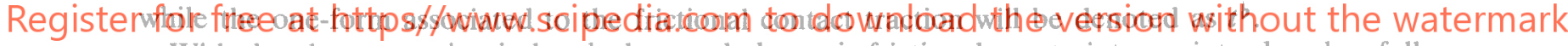

With the above notation in hand, the non-holonomic frictional, constraints are introduced as follows:

(i) Slip function. Admissible tractions space. We define a slip function $\Phi: T_{v} S^{2} \times \mathbb{R}_{+} \rightarrow \mathbb{R}$ such that $\left(t_{\mathrm{T}}^{b}, t_{\mathrm{N}}\right) \in T_{\nu} S^{2} \times \mathbb{R}_{+}$are constrained to lie in the closed set of admissible tractions defined as

$$
\mathbb{E}_{t}:=\left\{\left(t_{\mathrm{T}}^{\mathrm{b}}, t_{\mathrm{N}}\right) \in T_{\nu} S^{2} \times \mathbb{R}_{+}: \Phi\left(t_{\mathrm{T}}^{\mathrm{b}}, t_{\mathrm{N}}\right) \leqslant 0\right\}
$$

In particular, for the classical Coulomb friction law the admissible traction space is defined by the slip function:

$$
\Phi\left(t_{\mathrm{T}}^{\mathrm{b}}, t_{\mathrm{N}}\right):=\left\|t_{\mathrm{T}}^{\mathrm{b}}\right\|-\mu t_{\mathrm{N}}
$$

where $\|\cdot\|$ denotes the norm of its argument and $\mu$ is the Coulomb friction coefficient.

(ii) Slip rule. The slip rule is defined as follows:

$$
\begin{array}{ll}
V_{\mathrm{T}}^{b}(X, t):=0 & \text { if } \Phi\left(t_{\mathrm{T}}^{\mathrm{b}}, t_{\mathrm{N}}\right)<0 \\
V_{\mathrm{T}}^{b}(X, t):=\gamma p_{\mathrm{T}}^{\mathrm{b}} & \text { if } \Phi\left(t_{\mathrm{T}}^{\mathrm{b}}, t_{\mathrm{N}}\right)=0
\end{array}
$$

where $p_{\mathrm{T}}^{b}:=\partial_{t_{\mathrm{T}}} \Phi\left(t_{\mathrm{T}}^{\mathrm{b}}, t_{\mathrm{N}}\right)$ and $\gamma \in \mathbb{R}_{+}$is the non-negative slip consistency factor. For the frictional Coulomb law $p_{T}^{b}$ is the normalized one-form frictional traction defined as $\boldsymbol{p}_{\mathrm{T}}^{b}:=t_{\mathrm{T}}^{\mathrm{b}} /\left\|\boldsymbol{t}_{\mathrm{T}}^{\mathrm{b}}\right\|$.

(iii) Slip consistency condition. The slip consistency condition states that the rate of change of the slip function must be zero for positive values of the slip consistency factor. Mathematically this condition is expressed as

$$
\gamma \dot{\Phi}\left(t_{\mathrm{T}}^{b}, t_{\mathrm{N}}\right)=0
$$

The above constraints set leads to the following non-holonomic constraint 


$$
V_{\mathrm{T}}^{\mathrm{b}}(X, t)=\gamma p_{\mathrm{T}}^{\mathrm{b}}
$$

subjected to the following Kuhn-Tucker complementarity and consistency conditions

$$
\begin{aligned}
& \phi\left(t_{\mathrm{T}}^{\mathrm{b}}, t_{\mathrm{N}}\right) \leqslant 0 \\
& \gamma \geqslant 0 \\
& \gamma \Phi\left(t_{\mathrm{T}}^{\mathrm{b}}, t_{\mathrm{N}}\right)=0 \\
& \gamma \Phi\left(t_{\mathrm{T}}^{\mathrm{b}}, t_{\mathrm{N}}\right)=0
\end{aligned}
$$

\subsection{The regularized problem}

The solution of the dynamics of a particle subjected to holonomic and non-holonomic constraints given by (2.8), (2.18) and (2.19) amounts to finding a solution within a constrained solution space. Different methods have been used to bypass this difficulty. Here, we will restrict to one of these methods, based on the regularization or penalization of the constraints.

(A) Regularized holonomic unilaterial constraints. The holonomic unilateral constraints from the impenetrability, non-adherence and contact persistency, are regularized introducing a normal penalty parameter $\epsilon_{\mathrm{N}}$ and substituting the Kuhn-Tucker complementarity conditions given in (2.8) by the following constitutive-like equation for the normal reaction:

$$
t_{\mathrm{N}}(X, t):=\epsilon_{\mathrm{N}}\left\langle g_{\mathrm{N}} \circ \varphi(X, t)\right\rangle
$$

$(2.20)$

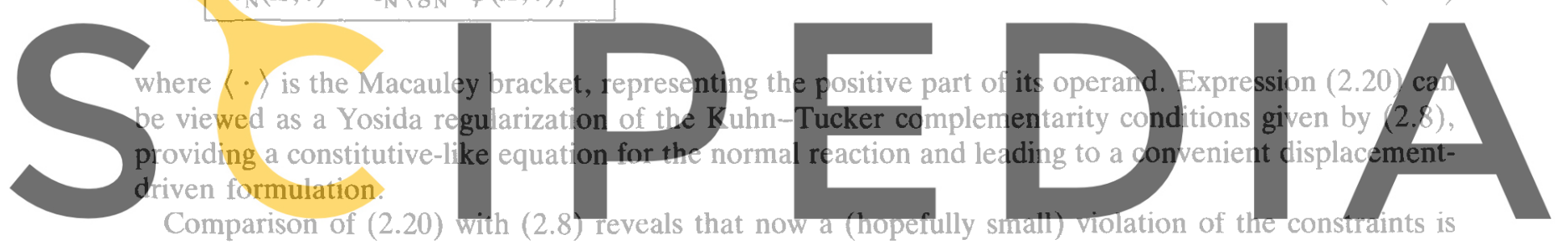

Comparison of (2.20) with (2.8) reveals that now a (hopefully smali) violation of the constraints is

allowed, and that the constraints will be exactly satisfied as $\epsilon_{\mathrm{N}} \rightarrow \infty$.

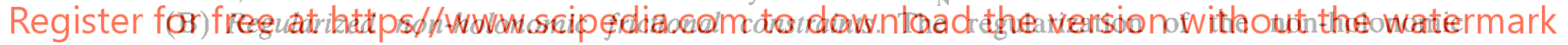

frictional constraints defined by (2.18) and (2.19) is defined introducing a tangential penalty parameter $\epsilon_{\mathrm{T}}$ playing the role of constitutive parameter in the slip velocity cvolution equation. Then the regularized non-holonomic frictional constraints take the form:

$$
\boldsymbol{V}_{\mathrm{T}}^{\mathrm{b}}(\boldsymbol{X}, t)=\gamma \boldsymbol{p}_{\mathrm{T}}^{\mathrm{b}}+\frac{1}{\epsilon_{\mathrm{T}}} \mathscr{L}_{V_{\mathrm{T}}} t_{\mathrm{T}}^{\mathrm{b}}
$$

subjected to the (non-regularized) Kuhn-Tucker complementarity conditions:

$$
\begin{aligned}
& \Phi\left(t_{\mathrm{T}}^{b}, t_{\mathrm{N}}\right) \leqslant 0 \\
& \gamma \geqslant 0 \\
& \gamma \Phi\left(t_{\mathrm{T}}^{b}, t_{\mathrm{N}}\right)=0 \\
& \gamma \Phi\left(t_{\mathrm{T}}^{\mathrm{b}}, t_{\mathrm{N}}\right)=0
\end{aligned}
$$

Here, $\mathscr{L}_{v_{T}} t_{\mathrm{T}}^{\mathrm{b}}$ is the Lie derivative of the frictional tangent traction along the flow induced by the slip velocity $\boldsymbol{V}_{\mathrm{T}}$.

Comparison of (2.21) with (2.18), reveals that the frictional constraints are exactly satisfied as $\epsilon_{\mathrm{T}} \rightarrow \infty$, in which case the (plastic) slip rate $\gamma$ is equal to the norm of the slip velocity $\boldsymbol{V}_{\mathrm{T}}^{\mathrm{b}}$. Otherwise, it is assumed that the slip velocity can be decomposed into an elastic or recoverable part and a plastic or irreversible part. Introduction of the Lie derivative in the regularized slip velocity, maintains frame indifference of the frictional evolution equations. 
(C) Frictional operator split. As we have seen above, the regularization of the constrained frictional problem leads to the following constrained frictional evolution equations:

$$
\begin{aligned}
& \mathscr{L}_{V_{\mathrm{T}}} t_{\mathrm{T}}^{b}=\epsilon_{\mathrm{T}}\left[V_{\mathrm{T}}^{b}-\gamma \partial_{t_{\mathrm{T}}^{b}} \Phi\left(t_{\mathrm{T}}^{\mathrm{b}}, t_{\mathrm{N}}\right)\right] \\
& \Phi\left(t_{\mathrm{T}}^{\mathrm{b}}, t_{\mathrm{N}}\right) \leqslant 0, \quad \gamma \geqslant 0, \quad \gamma \Phi\left(t_{\mathrm{T}}^{\mathrm{b}}, t_{\mathrm{N}}\right)=0 \\
& \gamma \dot{\Phi}\left(t_{\mathrm{T}}^{\mathrm{b}}, t_{\mathrm{N}}\right)=0
\end{aligned}
$$

Within the context of the product formula algorithms, a frictional operator split of the constrained evolution problem can be introduced by means of a trial state, defined by freezing the irreversible (plastic) slip response, i.e. setting $\gamma=0$, as follows:

Problem 1: Trial state Problem 2. Return mapping

$$
\left\{\begin{array}{l}
\mathscr{L}_{V_{\mathrm{T}}} t_{\mathrm{T}}^{\mathrm{b}}:=\epsilon_{\mathrm{T}} \boldsymbol{V}_{\mathrm{T}}^{\mathrm{b}} \\
\text { unconstrained }
\end{array}\right\} \quad\left\{\begin{array}{l}
\mathscr{L}_{V_{\mathrm{T}}} t_{\mathrm{T}}^{\mathrm{b}}:=-\epsilon_{\mathrm{T}} \gamma \partial_{t_{\mathrm{T}}^{\mathrm{b}}} \Phi\left(t_{\mathrm{T}}^{\mathrm{b}}, t_{\mathrm{N}}\right) \\
\Phi\left(t_{\mathrm{T}}^{\mathrm{b}}, t_{\mathrm{N}}\right) \leqslant 0, \quad \gamma \geqslant 0, \quad \gamma \Phi\left(t_{\mathrm{T}}^{\mathrm{b}}, t_{\mathrm{N}}\right)=0
\end{array}\right\}
$$

Problem 1 is defined by an unconstrained evolution equation for the frictional traction, with initial conditions the same initial conditions of the original problem. Solution of Problem 1 leads to the trial state. Problem 2 is defined by a constrained evolution equation for the frictional traction, with initial conditions given by the solution of Problem 1.

Note, that problems associated to the choice of a local parametrization of the slip path, arise only in the trial state obtained as the solution of the Problem 1 induced by the operator split. To get the final state, obtained as the solution of Problem 2, a straight-forward return mapping algorithm can be

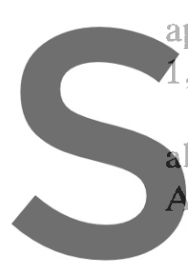
applied. Then, the focus ot the trial state. Problem 2 can be integrate ternatively using an IR n application of the gen

\subsection{Slip path definition. Computation of slip amount}

Register for free at https//www.scipedia.com to download the version without the watermark Time integration of the unconstrained evolution equations of Problem 1 given by (2.24), arising from an operator split and a product formula algorithm, involves the computation of the slip amount of the sliding particle onto the rigid surface. The key point of the new frictional time integration algorithm lies on the way in which Problem 1 is integrated and the slip amount is computed. Our goal here is to be able to compute the slip amount within a typical time interval, in terms of just the current placement of the particle and the unit outward normal to the surface at the beginning and at the end of the time interval. To avoid to compute the slip amount in terms of a local surface parametrization is a crucial point, when large slips are present and eventually the initial and final points of the slip path belongs to different local parametrization domains.

The main idea underlying the new frictional time integration algorithm is the following. The sliding velocity of the particle onto the rigid surface is integrated over a typical time interval, to get the incremental slip amount. This integration is performed by building an assumed slip path, carefully defined locally by the current placement of the particle and the unit outward normal to the surface at the beginning and at the end of the time step. Geometrically, this assumed slip path can be viewed as a second order approximation to the geodesic defined by these two points and their unit normals to the surface. Remarkably, the slip path is parametrized and the slip amount is computed without making any appeal to the underlying local surface parametrization, allowing large slip computations.

Consider the time interval of interest $I=[0, T]$ discretized into a series of non-overlapping subintervals $I:=\cup_{n=0}^{N}\left[t_{n}, t_{n+1}\right]$. Following a standard convention, we shall denote by either $(\cdot)_{n}$ or $(\cdot)_{n+1}$ the algorithmic approximations at times $t_{n}$ and $t_{n+1}$ to the continuum (time dependent) variable $(\cdot)_{t}$.

A step-by-step description of the slip path parametrization and the incremental slip amount computation within a typical subinterval $\left[t_{n}, t_{n+1}\right]$, is as follows: 
Step 1. Geometrical data. Consider given as initial conditions at time $t_{n}$, the current placement of the particle on the surface $Y_{n}:=\varphi\left(X, t_{n}\right)$ and the unit outward normal $\nu_{n}:=\nu \circ \varphi\left(X, t_{n}\right)$. Within the motion-driven framework, consider a prescribed motion of the particle from time $t_{n}$ to $t_{n+1}$, leading to its current placement on the surface denoted as $Y_{n+1}:=\varphi\left(X, t_{n+1}\right)$ and current unit outward normal denoted as $\nu_{n+1}:=\boldsymbol{\nu} \circ \varphi\left(X, t_{n+1}\right)$.

Step 2. Definition of the local orthonormal frames attached to $Y_{n}$ and $Y_{n+1}$, induced by the slip path. The crucial point of the new time integration algorithm lies on the construction and parametrization of the slip path as a second order approximation to the geodesic curve through the points $\boldsymbol{Y}_{n}$ and $\boldsymbol{Y}_{n+1}$ of the surface. Attached to the points $\boldsymbol{Y}_{n}$ and $\boldsymbol{Y}_{n+1}$ and induced by the slip curve, we define the set of orthonormal frames defined by the basis vectors $\left\{\boldsymbol{\nu}_{n}, \hat{\boldsymbol{\tau}}_{\alpha_{n}}\right\}$ and $\left\{\boldsymbol{\nu}_{n+1}, \hat{\boldsymbol{\tau}}_{\alpha_{n+1}}\right\}$, with $\alpha=1$, 2. Here, $\boldsymbol{\nu}_{n} \in S^{2}$ and $\boldsymbol{\nu}_{n+1} \in S^{2}$ are the unit outward normals to the surface at the points $\boldsymbol{Y}_{n}$ and $\boldsymbol{Y}_{n+1}$, respectively, $\hat{\tau}_{\alpha_{n}} \in T_{\nu_{n}} S^{2}$ and $\hat{\tau}_{\alpha_{n+1}} \in T_{\nu_{n+1}} S^{2}$ for $\alpha=1,2$ are unit tangent vectors to the surface at the points $Y_{n}$ and $\boldsymbol{Y}_{n+1}^{n}$, respectively, and $\hat{\tau}_{1_{n}} \in T_{\nu_{n}} S^{2}$ and $\hat{\tau}_{1_{n+1}} \in T_{\nu_{n+1}} S^{2}$ are defined in such a way that they are tangent to the slip curve at the points $\boldsymbol{Y}_{n}$ and $\boldsymbol{Y}_{n+1}$, respectively.

The definition of the local orthonormal frames $\left(\hat{\tau}_{1_{n}}, \hat{\tau}_{2_{n}}, \nu_{n}\right)$ and $\left(\hat{\tau}_{1_{n+1}}, \hat{\tau}_{2_{n+1}}, \boldsymbol{\nu}_{n+1}\right)$ attached to the points $\mathbb{Y}_{n}$ and $\mathbb{X}_{n+1}$, respectively, is as follows. Consider the Euclidean distance vector $d$ between the points $Y_{n}$ and $Y_{n+1}$, with Euclidean distance norm $d:=\|d\|$ and unit vector $\boldsymbol{m}$ defined as

$$
d=Y_{n+1}-Y_{n}, \quad d=\|d\|, \quad m=d / d
$$

Using the unit outward normals $\boldsymbol{\nu}_{n}$ and $\boldsymbol{\nu}_{n+1}$ and the unit vector $\boldsymbol{m}$ defined above, we construct two orthonormal frames as follows:
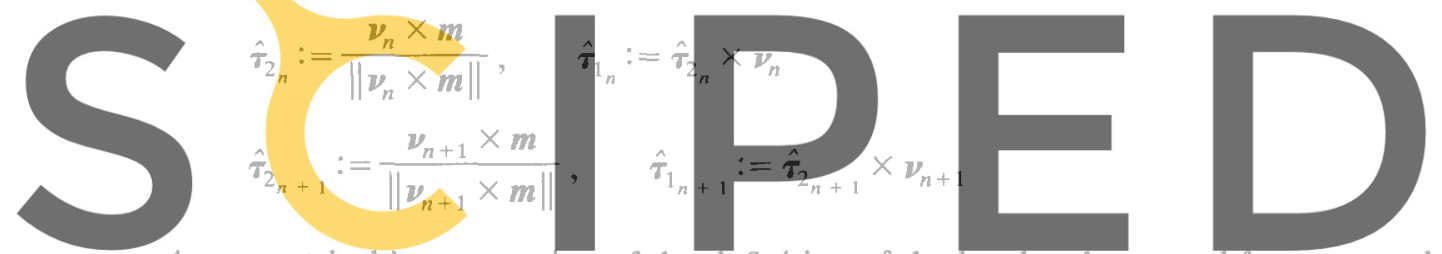

A geometrical interpretation of the definition of the local orthonormal frames attached to the initial and final slip path points is shown in Fig. 2.

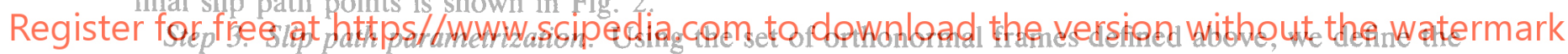

slip path as a second order approximation to the geodesic curve, via the map:

$$
\zeta \in[-1,+1] \mapsto \overline{\boldsymbol{Y}}(\zeta):=\mathscr{H}^{1}(\zeta) \boldsymbol{Y}_{u}+\frac{d}{2 \cos \Theta_{n}} \overline{\mathscr{H}}^{1}(\zeta) \hat{\tau}_{1_{n}}+\mathscr{H}^{2}(\zeta) \boldsymbol{Y}_{u+1}+\frac{d}{2 \cos \Theta_{n+1}} \overline{\mathscr{H}}^{2}(\zeta) \hat{\tau}_{1_{n+1}}
$$

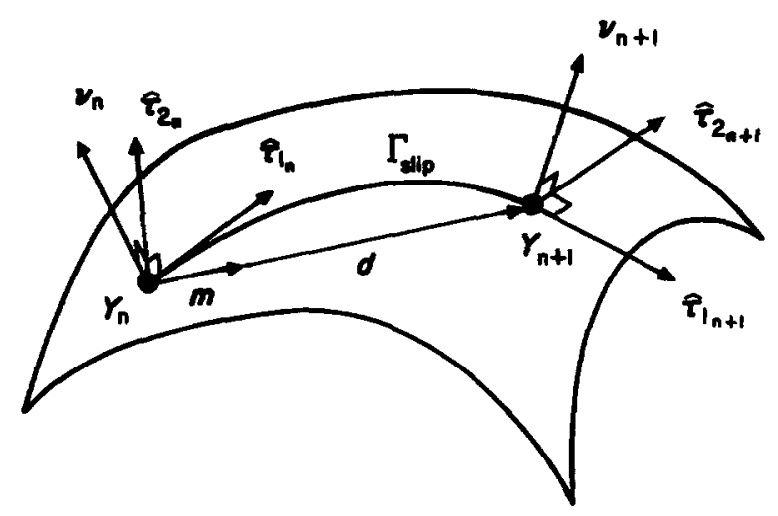

Fig. 2. Definition of lucal orthonormal frames attached to initial and final incremental slip path points. Parametrization of incremental slip path. 
where

$$
\begin{aligned}
& \cos \Theta_{n}:=\hat{\tau}_{1_{n}} \cdot \boldsymbol{m} \\
& \cos \Theta_{n+1}:=\hat{\tau}_{1_{n+1}} \cdot \boldsymbol{m}
\end{aligned}
$$

and $\mathscr{H}_{\alpha}, \overline{\mathscr{H}}_{\alpha}, \alpha=1,2$ are the Hermite shape functions defined in the isoparametric domain $[-1,1]$ as

$$
\begin{array}{ll}
\mathscr{H}^{1}(\zeta)=(2+\zeta)(1-\zeta)^{2} / 4, & \overline{\mathscr{H}}^{1}(\zeta)=(1+\zeta)(1-\zeta)^{2} / 4 \\
\mathscr{H}^{2}(\zeta)=(2-\zeta)(1+\zeta)^{2} / 4, & \overline{\mathscr{H}}^{2}(\zeta)=-(1-\zeta)(1+\zeta)^{2} / 4
\end{array}
$$

Step 4. Slip amount computation. The computation of the slip amount $\Gamma^{\text {slip }}$ is a follows. The tangent map to the curve defined by (2.27) takes the form:

$$
\zeta \in[-1,+1] \mapsto \overline{\boldsymbol{Y}}_{\mathrm{T}}(\zeta):=\nabla \mathscr{H}^{1}(\zeta) \boldsymbol{Y}_{n}+\frac{d}{2 \cos \Theta_{n}} \nabla \overline{\mathscr{H}}^{1}(\zeta) \hat{\boldsymbol{\tau}}_{1_{n}}
$$

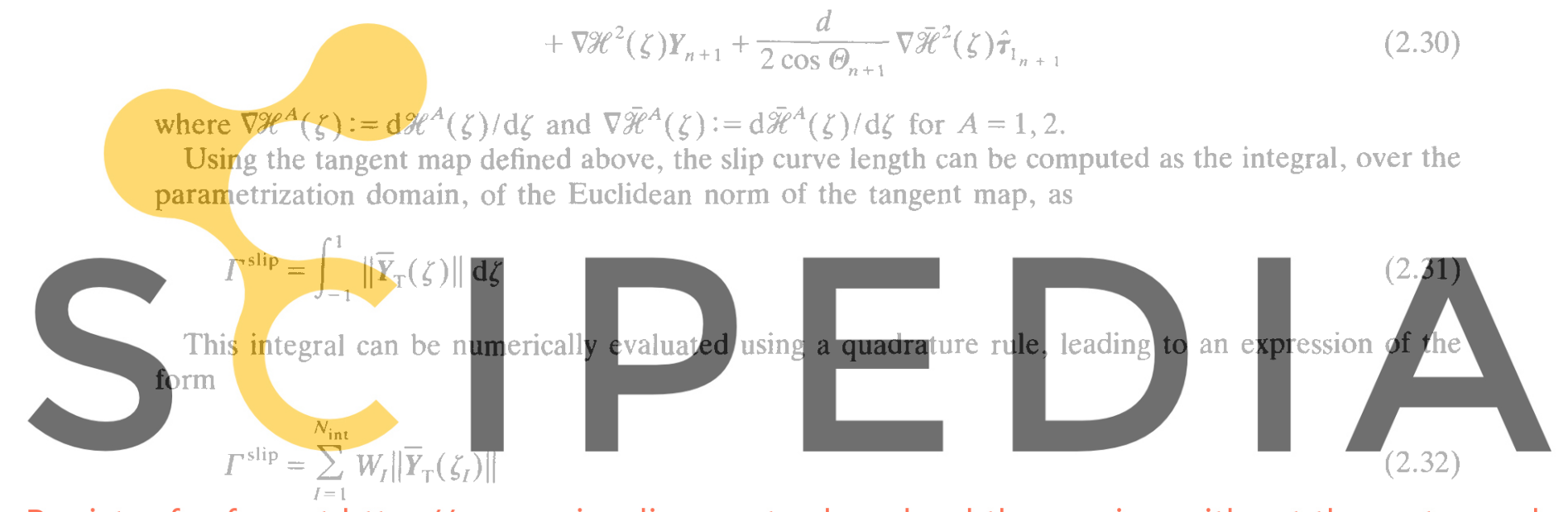

Register for free at https//www. scipedia, com to downdoad the version without the watermark are the integration weights and $N_{\text {int }}$ is the number of integration points to be used in the quadrature rule.

\section{A new time integration algorithm for the non-holonomic frictional constrained evolution problem}

In this section we present a new frictional time-stepping algorithm for the time integration of the non-holonomic frictional constrained evolution problem. To focus on the main idea of the algorithm, it will be introduced within the context of the simple model problem described in Section 2 . The algorithm is amenable to exact linearization, leading to an asymptotic quadratic rate of convergence when used within an iterative Newton-Raphson solution scheme. The algorithm is suitable for large slip multi-body frictional contact problems.

As we have seen above, the new frictional time integration algorithm focussed on the solution of Problem 1, arising from the operator split (2.24), while Problem 2 can be integrated using a frictional return mapping algorithm.

The main idea underlying the new integration algorithm is the following. The sliding velocity of the particle is integrated to get the slip amount. The procedure to define the slip path parametrization and the algorithm used to compute the slip amount has been shown in Section 2.3. As mentioned earlier, this is the key point of the new frictional time integration algorithm. On the other hand, the Lie derivative along the flow induced by the slip velocity, arising in Problem 1 in (2.24), is integrated by means of a shifter or orthogonal parallel transport, along the slip path, operator.

Integration of the constrained evolution equations (2.24) in Problem 2, which takes place at a fixed 
configuration, is performed by a straightforward application of a frictional return mapping algorithm. As this integration takes place at a fixed configuration, the local surface parametrization can be used.

Consider the time interval of interest $I=[0, T]$ discretized into a series of non-overlapping subintervals $I:=\cup_{n=0}^{N}\left[t_{n}, t_{n+1}\right]$. The incremental solution to the frictional constrained evolution problem defined by (2.23), is obtained applying a time stepping algorithm to integrate the evolution equations within a typical time step $\left[t_{n}, t_{n+1}\right]$ with given states variables, as initial conditions, at time $t_{n}$.

Following a standard convention, we shall denote by either $(\cdot)_{n}$ or $(\cdot)_{n+1}$ the algorithmic approximations at times $t_{n}$ and $t_{n+1}$ to the continuum (time dependent) variable $(\cdot)_{t}$.

A step-by-step description of the new frictional time integration algorithm is as follows:

Step 1. Initial conditions at time $t_{n}$ : Database. Consider given as initial conditions at time $t_{n}$, the frictional traction $t_{T_{n}}^{b}$, the placement of the particle on the surface $Y_{n}:=\varphi\left(X, t_{n}\right)$ and the unit outward normal $\nu_{n}:=\nu \circ \varphi\left(X, t_{n}\right)$. Within the motion-driven framework, consider a prescribed motion of the particle from time $t_{n}$ to $t_{n+1}$, leading to its current placement on the surface denoted as $Y_{n+1}:=$ $\boldsymbol{\varphi}\left(\boldsymbol{X}, t_{n+1}\right)$ and current unit outward normal denoted as $\nu_{n+1}:=\boldsymbol{\nu} \circ \boldsymbol{\varphi}\left(\boldsymbol{X}, t_{n+1}\right)$.

Step 2. Definition of the local orthonormal frames attached to $\mathbb{X}_{n}$ and $\boldsymbol{X}_{n+1}$, induced by the slip path. Using the unit outward normals $\nu_{n} \in S^{2}$ and $\nu_{n+1} \in S^{2}$ and (2.25) we define the unit tangent vectors $\hat{\tau}_{\alpha_{n}} \in T_{\nu} S^{2}$ and $\hat{\tau}_{\alpha_{n+1}} \in T_{v_{n+1}} S^{2}$ given by (2.26). The unit outward normals together with the unit tangent yectors define two set of orthonormal basis $\left(\boldsymbol{\nu}_{\mu}, \hat{\tau}_{1}, \hat{\tau}_{2}\right) \in S^{2} \times T_{\nu} S^{2} \times T_{\nu} S^{2}$ and $\left(\nu_{n+1}, \hat{\tau}_{1_{n+1}}, \hat{\tau}_{2_{n+1}}\right) \in S^{2} \times T_{\nu_{n+1}} S^{2} \times T_{\nu_{n+1}} S^{2}$, attached to the points $\boldsymbol{Y}_{n}$ and $\boldsymbol{Y}_{n+1}$, respectively.

Step 3. Slip path parametrization and slip amount computation. Using the set of orthonormal basis defined above, we define a slip path via the map (2.27). The tangent map (2.30) is used to define the slip amount given by (2.31). Slip amount can be numerically computed, using a quadrature rule to

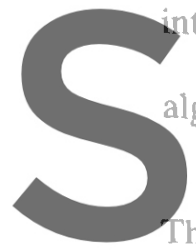
nitegrate (2.31), leading to Step 4. Trial frictional lgorithm:

(i) Parallel transport

(ii) Trial slip contributio
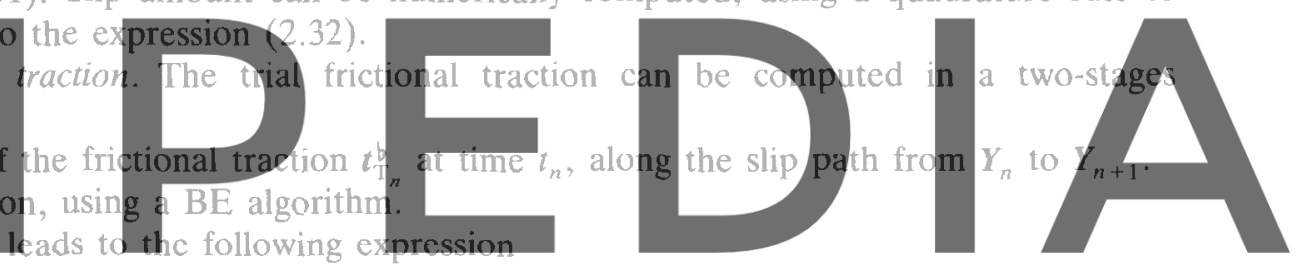

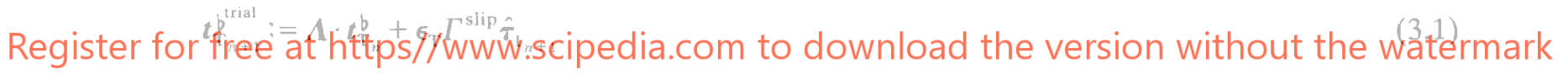

where $\Gamma_{\text {slip }}$ is the slip amount, $\Lambda \in \mathrm{SO}(3)$ is the shifter or orthogonal parallel transport operator defined as

$$
\Lambda:=\hat{\tau}_{\alpha_{n+1}} \otimes \hat{\tau}_{\alpha_{n}}, \quad \alpha=1,2
$$

where $\mathrm{SO}(3)$ is the special orthogonal group defined as

$$
\operatorname{SO}(3):=\left\{\boldsymbol{\Lambda}: \mathbb{R}^{3} \rightarrow \mathbb{R}^{3} \mid \boldsymbol{\Lambda}^{\mathfrak{\Upsilon}}=\boldsymbol{\Lambda}^{-1} \text { and } \operatorname{det}[\boldsymbol{\Lambda}]=+1\right\}
$$

Expression (3.1) represents the semi-discrete counterpart of the evolution problem given by the trial state in (2.24), where the shifter or parallel transport operator has been introduced to integrate the Lie derivative along the flow induced by the slip velocity and a Backward-Euler time stepping algorithm has been used to integrate the slip velocity.

Step 5. Projection onto the Coulomb disk. Return mapping algorithm. The computed trial frictional traction on current configuration is projected onto the Coulomb disk via a return mapping algorithm. This projection, involves the computation of the trial slip function defined as

$$
\Phi_{n+1}^{\text {trial }}:=\left\|t_{T_{n+1}^{\text {trial }}}^{\text {tri }}\right\|-\mu t_{N_{n+1}}
$$

If $\Phi_{n+1}^{\text {trial }} \leqslant 0$ then the trial frictional traction satisfies the frictional constraints and the projection is just

$$
\boldsymbol{t}_{\mathrm{T}_{n+1}}^{\mathrm{b}}:=\boldsymbol{t}_{\mathrm{T}_{n+1}}^{\mathrm{trial}^{\mathrm{tial}}}
$$

otherwise, a projection of the trial values onto the Coulomb disk must be performed. For the simple 
Table 1

Frictional time integration algorithm

1. Initial conditions at time $t_{n}:$ Database. Given the history $\left\{t_{T_{n}}^{b}, Y_{n}, \nu_{n}\right\}$ for the (one form) frictional traction, cartesian coordinates and unit outward normal (at the slave quadrafure points) and for prescribed (motion driven) values $\left\{\boldsymbol{Y}_{n+1}, \boldsymbol{\nu}_{n+1}\right\}$

2. Definition of local orthonormal frames attached to $Y_{n}$ and $Y_{n+1}$ induced by the slip path.

Set

$$
\begin{aligned}
& \hat{\boldsymbol{\tau}}_{2_{n}}:=\frac{\boldsymbol{\nu}_{\boldsymbol{n}} \times \boldsymbol{m}}{\left\|\boldsymbol{\nu}_{n} \times \boldsymbol{m}\right\|}, \quad \hat{\boldsymbol{\tau}}_{1_{n}}:=\hat{\boldsymbol{\tau}}_{2_{n}} \times \boldsymbol{\nu}_{n} \\
& \hat{\boldsymbol{\tau}}_{2_{n+1}}:=\frac{\boldsymbol{\nu}_{n+1} \times \boldsymbol{m}}{\left\|\boldsymbol{\nu}_{n+1} \times \boldsymbol{m}\right\|}, \quad \hat{\boldsymbol{\tau}}_{1_{n+1}}:=\hat{\boldsymbol{\tau}}_{2_{n+1}} \times \boldsymbol{\nu}_{n+1}
\end{aligned}
$$

where

$$
\boldsymbol{d}=\boldsymbol{Y}_{n+1}-\mathbf{Y}_{n}, \quad d=\|d\|, \quad \boldsymbol{m}=\boldsymbol{d} / d
$$

3. Slip path parametrization and slip amount.

(i) Slip path parametrization

$$
\begin{aligned}
\zeta \in[-1,+1] \mapsto \bar{Y}(\zeta):= & \mathscr{H}^{1}(\zeta) Y_{n}+\frac{d}{2 \cos \Theta_{n}} \overline{\mathscr{H}}^{1}(\zeta) \hat{\boldsymbol{\tau}}_{1_{n}} \\
& +\mathscr{H}^{2}(\zeta) \boldsymbol{Y}_{n+1}+\frac{d}{2 \cos \Theta_{n+1}} \overline{\mathscr{H}}^{2}(\zeta) \hat{\tau}_{1_{n+1}}
\end{aligned}
$$

where

$$
\begin{array}{ll}
\cos \Theta_{n}:=\hat{\tau}_{1_{n}} \cdot \boldsymbol{m}, \quad \cos \Theta_{n+1}:=\hat{\tau}_{1_{n+1}} \cdot \boldsymbol{m} \\
\mathscr{H}^{1}(\zeta)=(2+\zeta)(1-\zeta)^{2} / 4, & \overline{\mathscr{H}}^{1}(\zeta)=(1+\zeta)(1-\zeta)^{2} / 4 \\
\mathscr{H}^{2}(\zeta)=(2-\zeta)(1+\zeta)^{2} / 4, & \overline{\mathscr{H}}^{2}(\zeta)=-(1-\zeta)(1+\zeta)^{2} / 4
\end{array}
$$

(ii) Tangent map

$$
\begin{aligned}
\zeta \in[-1,+1] \mapsto \overline{\boldsymbol{Y}}_{\mathrm{T}}(\zeta):= & \nabla \mathscr{H}^{1}(\zeta) \boldsymbol{Y}_{n}+\frac{d}{2 \cos \Theta_{n}} \nabla \overline{\mathscr{H}}^{1}(\zeta) \hat{\tau}_{1_{n}} \\
& +\nabla \mathscr{H}^{2}(\zeta) \boldsymbol{Y}_{n+1}+\frac{d}{2 \cos \Theta_{n+1}} \nabla \overline{\mathscr{H}}^{2}(\zeta) \hat{\tau}_{1_{n+1}}
\end{aligned}
$$

(iii) Slip amount

$$
\Gamma^{\text {slip }}=\sum_{I=1}^{N_{\mathrm{int}}} W_{l}\left\|\overline{\boldsymbol{Y}}_{\mathrm{r}}\left(\zeta_{J}\right)\right\|
$$

4. Trial state.

(i) Trial frictional traction

$$
t_{\mathrm{T}_{n+1}}^{\text {trial }}:=\boldsymbol{\Lambda} \cdot t_{\mathrm{T}_{n}}^{b}+\epsilon_{\mathrm{T}} \Gamma^{\mathrm{slip}} \hat{\tau}_{1_{n+1}}
$$

where

$$
\boldsymbol{\Lambda}:=\hat{\boldsymbol{\tau}}_{\alpha_{n+1}} \otimes \hat{\boldsymbol{\tau}}_{a_{n}}, \quad \alpha=1,2
$$

(ii) Trial slip function

$$
\Phi_{n+1}^{\text {trial }}:=\left\|t t_{T_{n+1}}^{\text {trial }}\right\|-\mu t_{N_{n+1}}
$$

5. Frictional return mapping: Projection onto the Coulomb disk.

(i) Return mapping

$$
t_{\mathrm{T}_{n+1}}= \begin{cases}t_{\mathrm{T}_{n+1}}^{\text {trial }} & \text { if } \Phi_{n+1}^{\text {trial }} \leqslant 0 \text { (stick) } \\ \mu t_{\mathrm{N}_{n+1}} p_{\mathrm{T}_{n+1}}^{\text {trial }} & \text { otherwise (slip) }\end{cases}
$$

where

$$
\boldsymbol{p}_{\mathrm{T}_{n+1}}^{b_{\text {trial }}}=\boldsymbol{t}_{\mathrm{T}_{n+1}}^{\mathrm{b}_{\text {trial }}} /\left\|\boldsymbol{t}_{\mathrm{T}_{n+1}}^{\boldsymbol{b}_{\text {trial }}}\right\|
$$


classical Coulomb model, the return mapping algorithm leads to a direct computation of the frictional tangent traction given by

$$
\boldsymbol{t}_{T_{n+1}}^{\mathrm{b}}:=\mu t_{\mathrm{N}_{n+1}} p_{\mathrm{T}_{n+1}}^{b^{\text {trial }}}
$$

where $\boldsymbol{p}_{\mathrm{T}_{n+1}}^{\text {trial }}=\boldsymbol{t}_{\mathrm{T}_{n+1}}^{\text {trial }^{\text {tial }}} /\left\|\boldsymbol{t}_{\mathrm{T}_{n+1}}^{\mathbf{t}^{\text {trial }}}\right\|$.

A summary of the frictional time integration algorithm is shown in Table 1.

REMARK 3.1. The whole algorithm has been introduced within the simple context of the model problem of a particle subjected to holonomic unilateral constraints, arising from the presence of a rigid obstacle, and to non-holonomic frictional constraints, arising from the frictional roughness behaviour at the surface. The extension of the algorithm to a finite deformation, large slip multi-body frictional contact framework is straightforward. Within this general framework, the computation of the trial frictional traction is performed at a fixed reference configuration. Once the trial frictional traction on reference configuration has been computed, one must perform its push-forward to the current configuration and then perform the projection onto the Coulomb disk via a return mapping algorithm on current configuration.

REMARK 3.2. The algorithm depicted above is amenable to exact linearization leading to a consistent or algorithmic tangent operator. The linearization of the algorithm, within the general context of finite deformation, large slip multi-body frictional contact problems, is included in Appendix B.

REMARK 3.3. The implementation of the new frictional time integration algorithm within an Augmented Lagrangian method is straightforward. In this case it would be necessary to build up a new slip path in each one of the nested iterations of the Lagrange multipliers augmentations.

\section{Numerical simulations}

The formulation presented in the preceding sections is illustrated below in a number of full three-dimensional numerical simulations. The goals are to show the performance of the new frictional contact time integration algorithm at large slips and finite deformations and to demonstrate the robustness of the overall finite element formulation in different numerical analyses. The calculations are performed with an enhanced version of the finite element program FEAP developed by R.L. Taylor and J.C. Simo and documented in [24].

(A) Forming of an oil pan. This example is taken from Laursen and Simo [12] and provides an illustration of an industrial application of the preceding theory. An initially flat elastoplastic metal sheet is formed into a pan, by forcing it to conform to the shape of a rigid punch pressed against it. The sheet is considered to be clamped at the edges to a fixed rigid die. A $J_{2}$-model of multiplicative plasticity is assumed, with material properties $E=70 \mathrm{GPa}, \nu=0.3, \sigma_{Y}=140 \mathrm{MPa}$ and isotropic hardening with hardening modulus $H=100 \mathrm{MPa}$. A Coulomb friction model for the contact interface between the rigid tools and the sheet is assumed with a friction coefficient of 0.25 . The sheet initially measured $600 \mathrm{~mm}$ long, $560 \mathrm{~mm}$ wide and $5 \mathrm{~mm}$ thick. The punch consist of a lower flat region with an inclined section leading to it. The forming process was continued until the lower region had been moved through a distance of $100 \mathrm{~mm}$.

For symmetry reasons only half the geometry was modeled, with 800 continuum elements being utilized for the discretization of the sheet. A mixed Q1/P0 finite element formulation at finite strain, with piece-wise constant interpolations for both pressure and volume along with a bilinear interpolation for the displacement field has been used. See, for example, Simo [22] (Section 45), for a description of the method. The penalty parameters for contact and friction have been taken as $\varepsilon_{\mathrm{N}}=\varepsilon_{\mathrm{T}}=10^{10}$ and the new frictional time integration algorithm has been used in the simulation. The loading was achieved in 100 load steps, through displacement control of the punch. The Newton-Raphson method, combined 
Table 2

Forming of an oil pan. Euclidean residual norm for four typical time steps

\begin{tabular}{llll}
\hline Step 25 & Step 50 & Step 75 & Step 100 \\
\hline $2.72291 \mathrm{E}+04$ & $2.99612 \mathrm{E}+04$ & $3.31514 \mathrm{E}+04$ & $3.09564 \mathrm{E}+04$ \\
$7.24502 \mathrm{E}+04$ & $7.95762 \mathrm{E}+04$ & $6.04478 \mathrm{E}+04$ & $5.94460 \mathrm{E}+04$ \\
$1.63322 \mathrm{E}+04$ & $1.87194 \mathrm{E}+04$ & $8.44338 \mathrm{E}+04$ & $3.50416 \mathrm{E}+04$ \\
$1.57428 \mathrm{E}+03$ & $2.38115 \mathrm{E}+03$ & $6.17215 \mathrm{E}+03$ & $2.21297 \mathrm{E}+03$ \\
$1.60151 \mathrm{E}+02$ & $1.59523 \mathrm{E}+02$ & $1.22857 \mathrm{E}+02$ & $5.76473 \mathrm{E}+01$ \\
$1.12800 \mathrm{E}+01$ & $2.08237 \mathrm{E}+00$ & $2.29146 \mathrm{E}+01$ & $1.50948 \mathrm{E}+00$ \\
$4.32125 \mathrm{E}-03$ & $2.50423 \mathrm{E}-04$ & $5.38027 \mathrm{E}-01$ & $1.34566 \mathrm{E}-04$ \\
$6.46626 \mathrm{E}-08$ & $6.48886 \mathrm{E}-08$ & $2.12824 \mathrm{E}-06$ & $1.38417 \mathrm{E}-07$ \\
\hline
\end{tabular}

with a line search optimization procedure, has been used to solve the nonlinear system of equations arising from the spatial and temporal discretization.

The analysis was performed in a Silicon Graphics Power Challenge L. Workstation and it was accomplished in $2 \mathrm{~h} 31 \mathrm{~min}$ CPU time. Table 2 summarizes the values of the Euclidean norm of the residual obtained for four typical time steps, corresponding to $25,50,75$ and $100 \mathrm{~mm}$ punch displacement, in an iterative solution procedure employing Newton's method. The quadratic rate of convergence exhibited by the iteration is the result of an exact linearization of the problem, including frictional contact constraints, leading to an exact expression for the algorithmic tangent moduli.
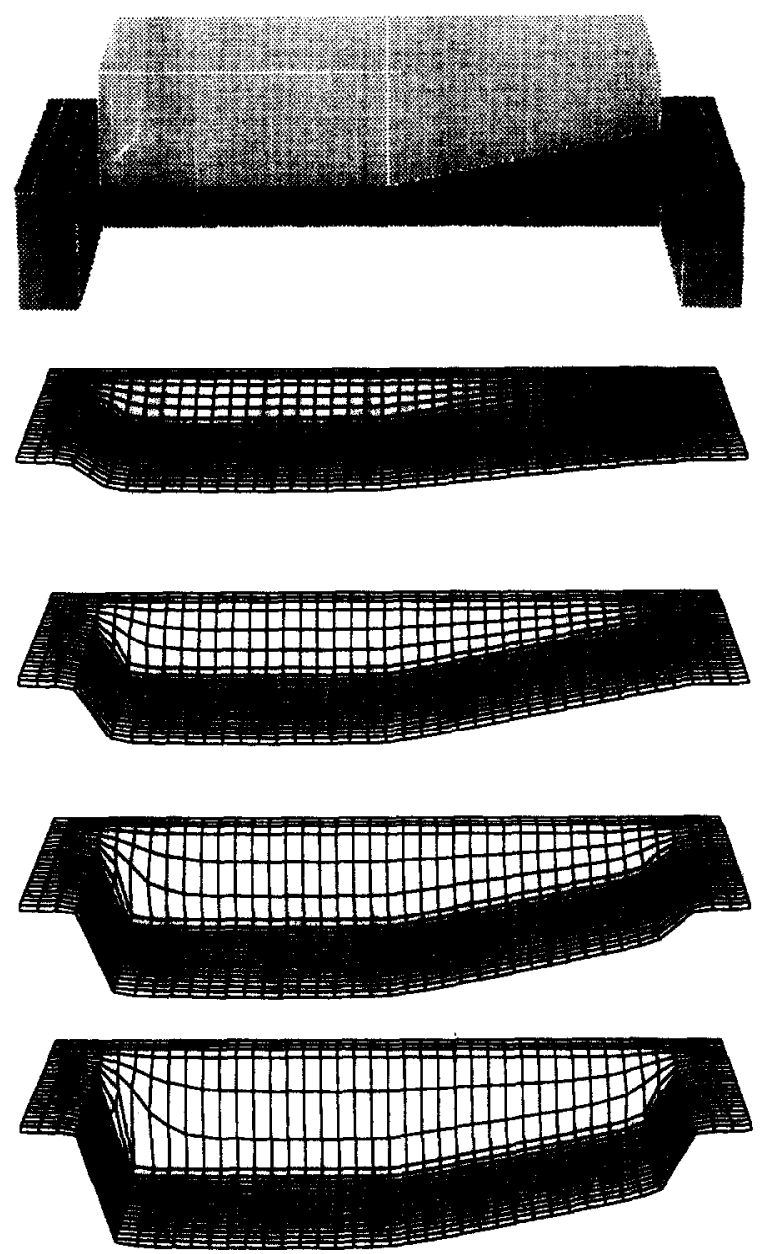

Fig. 3. Forming process of an oil pan. Initial configuration for the sheet and tools and deformed shapes at $25,50,75$ and $100 \mathrm{~mm}$ punch displacement of the forming process. 

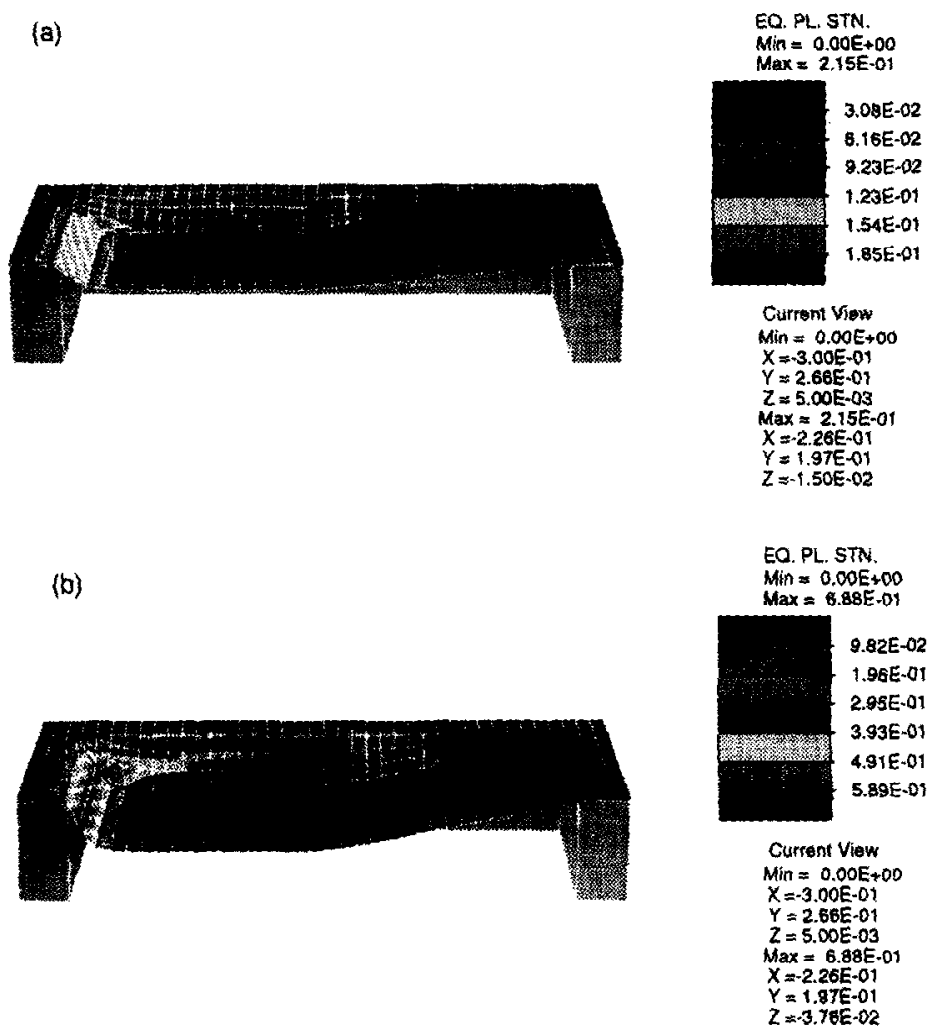

(c)
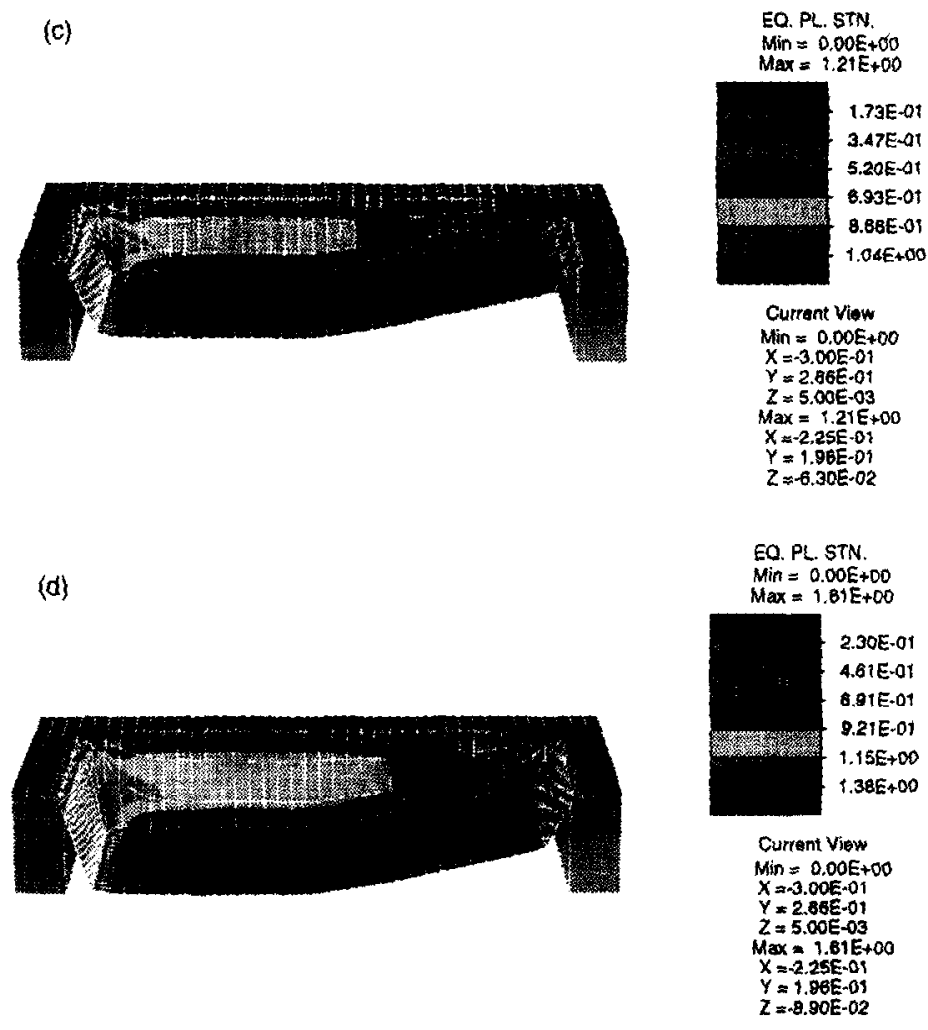

Fig. 4. (a) Forming process of an oil pan. Cuntour of equivalent plastic strain at a punch displacement of $25 \mathrm{~mm}$. (b) Forming process of an oil pan. Contour of equivalent plastic strain at a punch displacement of $50 \mathrm{~mm}$. (c) Forming process of an oil pan. Contour of equivalent plastic strain at a punch displacement of $75 \mathrm{~mm}$. (d) Forming process of an oil pan. Contour of equivalent plastic strain at the final punch displacement of $100 \mathrm{~mm}$. 

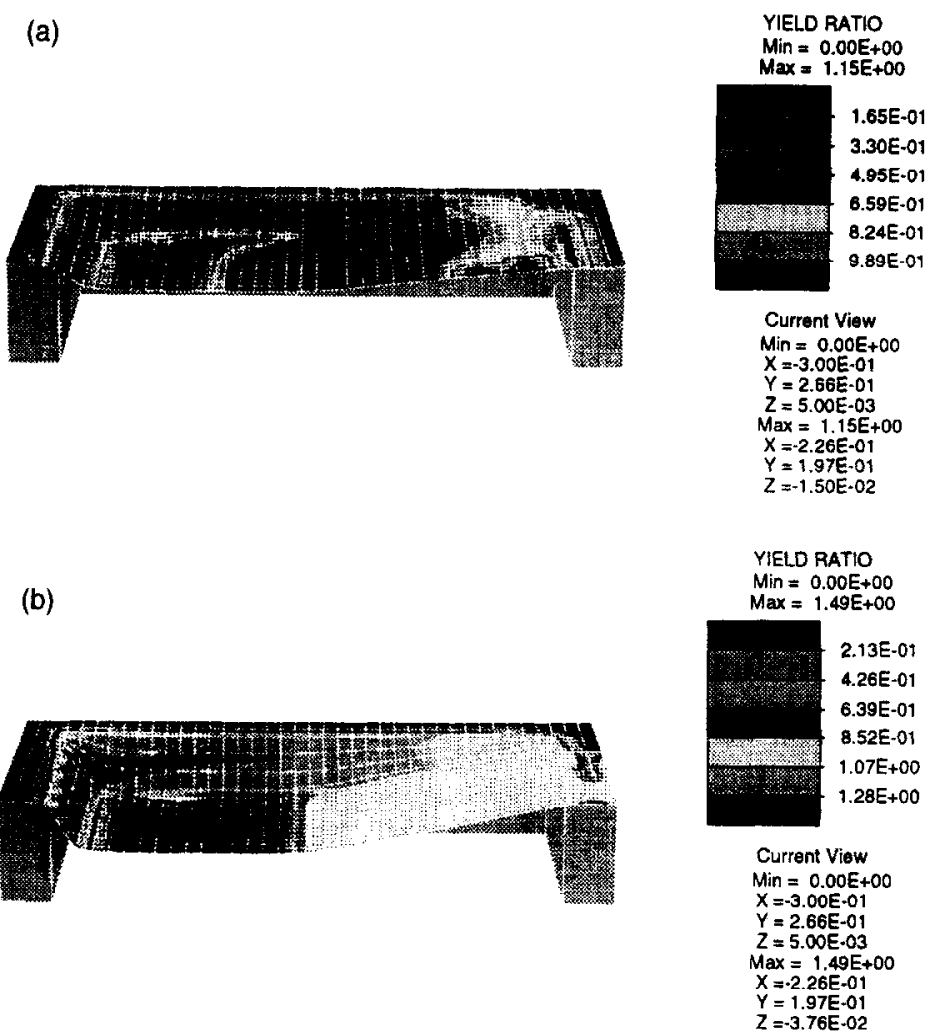

(d)
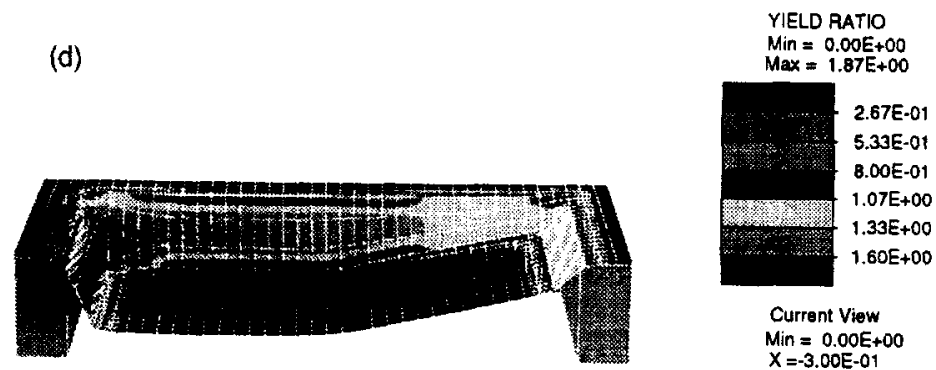

Current View

$\operatorname{Min}=0.00 \mathrm{E}+00$

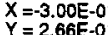

$Z=5.00 \mathrm{E}-03$

$\operatorname{Max}=1.87 E+00$

$X=-2.25 E-01$

$Y=1.96 \mathrm{E}-01$
$Z=-6.30 \mathrm{E}-02$

(d)

YIELD RATIO

$M$ Max $=2.00 E+00$
Mat $+\infty 0$
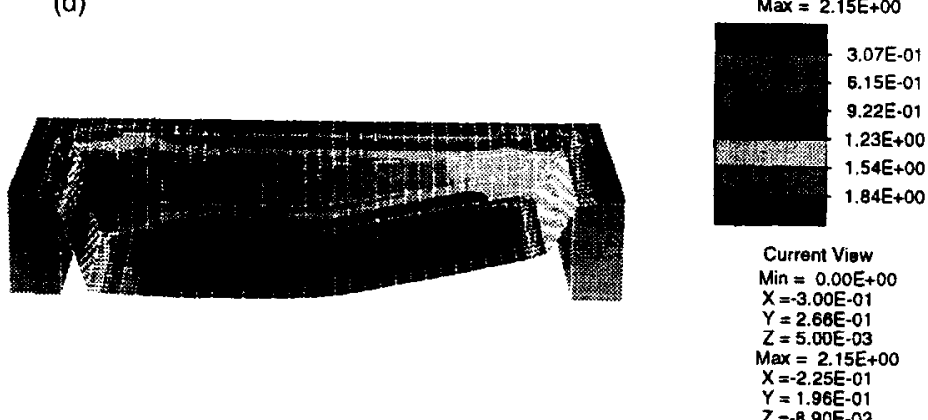

Fig. 5. (a) Forming process of an oil pan. Contour of yield ratio at a punch displacement of $25 \mathrm{~mm}$. (b) Forming process of an oil pan. Contour of yield ratio at a punch displacement of $50 \mathrm{~mm}$. (c) Forming process of an oil pan. Contour of yield ratio at a punch displacement of $75 \mathrm{~mm}$. (d) Forming process of an oil pan. Contour of yield ratio at the final punch displacement of $100 \mathrm{~mm}$. 
Fig. 3 shows the initial configuration of the sheet and the tools, punch and die, along with the deformed shapes of the sheet at four different stages of the forming process, corresponding to punch displacements of $25,50,75$ and $100 \mathrm{~mm}$. Figs. 4 and 5 show, respectively, the contours of equivalent plastic strain and yield ratio at the same four different stages of the forming process.

(B) Pinching of a pipe by two flexible plates. This example is taken from Laursen and Simo [11] and is concerned with the pinching of an aluminium pipe by a pair of aluminium plates. The material properties for both the pipe and the two plates were taken as bulk modulus $K=74.4 \mathrm{GPa}$, shear modulus $G=28.5 \mathrm{GPa}$ and yield stress $\sigma_{Y}=485 \mathrm{MPa}$. No hardening effects were assumed. The pipe had an inner radius of $9 \mathrm{~cm}$, an outer radius of $10 \mathrm{~cm}$ and a length of $40 \mathrm{~cm}$. The plates were each one $2 \mathrm{~cm}$ thick and measured $40 \mathrm{~cm}$ length by $15 \mathrm{~cm}$ wide. A Coulomb friction coefficient of $\mu=0.1$ was assumed between the plates and the pipe.

Due to symmetry only one-eighth of the geometry was modeled with 128 continuum elements being utilized for the discretization of the pipe and 15 continuum elements for the discretization of each of the plates. A mixed Q1/P0 finite element formulation at finite strains, with piece-wise constant interpolations for both pressure and volume along with a bilinear interpolation for the displacement field, has been used. See, for example, Simo [22] (Section 45), for a description of the method. The penalty parameters for contact and friction have been taken as $\varepsilon_{\mathrm{N}}=\varepsilon_{\mathrm{T}}=10^{10}$. The new frictional time integration algorithm has been used in the simulation. The loading was achieved in 100 load steps, through displacement control of the shorter edges of the plates. The Newton-Raphson method, combined with a line search optimization procedure, has been used to solve the nonlinear system of equations arising from the spatial and temporal discretization.

The analysis was performed in a Silicon Graphics Challenge L Workstation and it was accomplished in 27 min CPU time. Table 3 shows the Euclidean norm of the residual at four typical time steps.

This example demonstrates the capability of the proposed formulation to handle problems in which different finite deformable bodies are involved. Fig. 6 shows the geometry of the problem at the initial configuration along with the deformed shape at four different stages of the process. Figs. 7 and 8 show the contour of equivalent plastic strain and yield ratio, respectively, at these four stages of the pinching process. Fig. 9 shows the equivalent plastic strain contours at the final stage. Here, half the geometry is
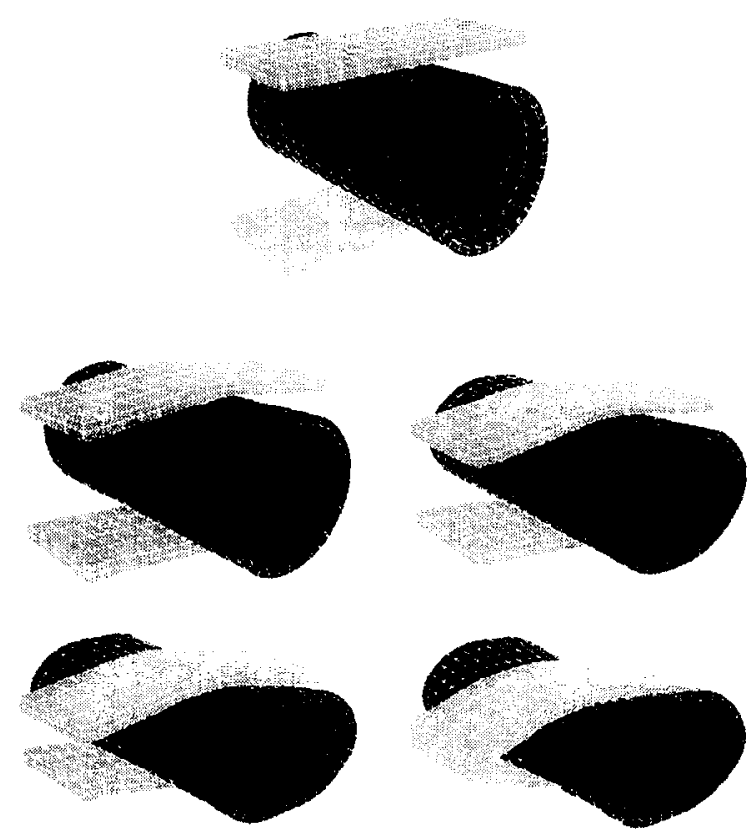

Fig. 6. Pinching of a pipe by two flexible plates. Initial configuration for the sheet and tools and deformed shapes at $25,50,75$ and $100 \mathrm{~mm}$ plate edges displacement. 
Table 3

Pinching of a pipe by two flexible plates. Euclidean residual norm for four typical time steps

\begin{tabular}{llll}
\hline Step 25 & Step 50 & Step 75 & Step 100 \\
\hline $8.11798 \mathrm{E}+09$ & $1.12212 \mathrm{E}+10$ & $1.02522 \mathrm{E}+10$ & $9.94982 \mathrm{E}+09$ \\
$8.48452 \mathrm{E}+07$ & $4.69798 \mathrm{E}+08$ & $6.32628 \mathrm{E}+08$ & $3.16392 \mathrm{E}+09$ \\
$2.06944 \mathrm{E}+06$ & $8.54720 \mathrm{E}+07$ & $6.33897 \mathrm{E}+08$ & $1.50084 \mathrm{E}+09$ \\
$2.05370 \mathrm{E}+04$ & $1.42299 \mathrm{E}+07$ & $1.74784 \mathrm{E}+07$ & $5.68297 \mathrm{E}+08$ \\
$5.81648 \mathrm{E}+00$ & $7.20342 \mathrm{E}+04$ & $2.33905 \mathrm{E}+06$ & $5.20430 \mathrm{E}+07$ \\
$8.62700 \mathrm{E}-04$ & $4.33376 \mathrm{E}+00$ & $6.22580 \mathrm{E}+02$ & $4.68104 \mathrm{E}+06$ \\
& $9.95670 \mathrm{E}-04$ & $8.67621 \mathrm{E}-04$ & $9.75066 \mathrm{E}+04$ \\
& & & $2.44372 \mathrm{E}+00$ \\
& & & $9.69764 \mathrm{E}-04$ \\
\hline
\end{tabular}

represented to better appreciate the final deformed shape of the pipe at the middle section as well as the distribution of the equivalent plastic strain at the middle inner part of the pipe.

(C) Draw Bead Simulator. This example is concerned with the simulation of a draw bead in a deep drawing sheet metal forming process. An initially flat strip metal sheet will be drawn through a set of
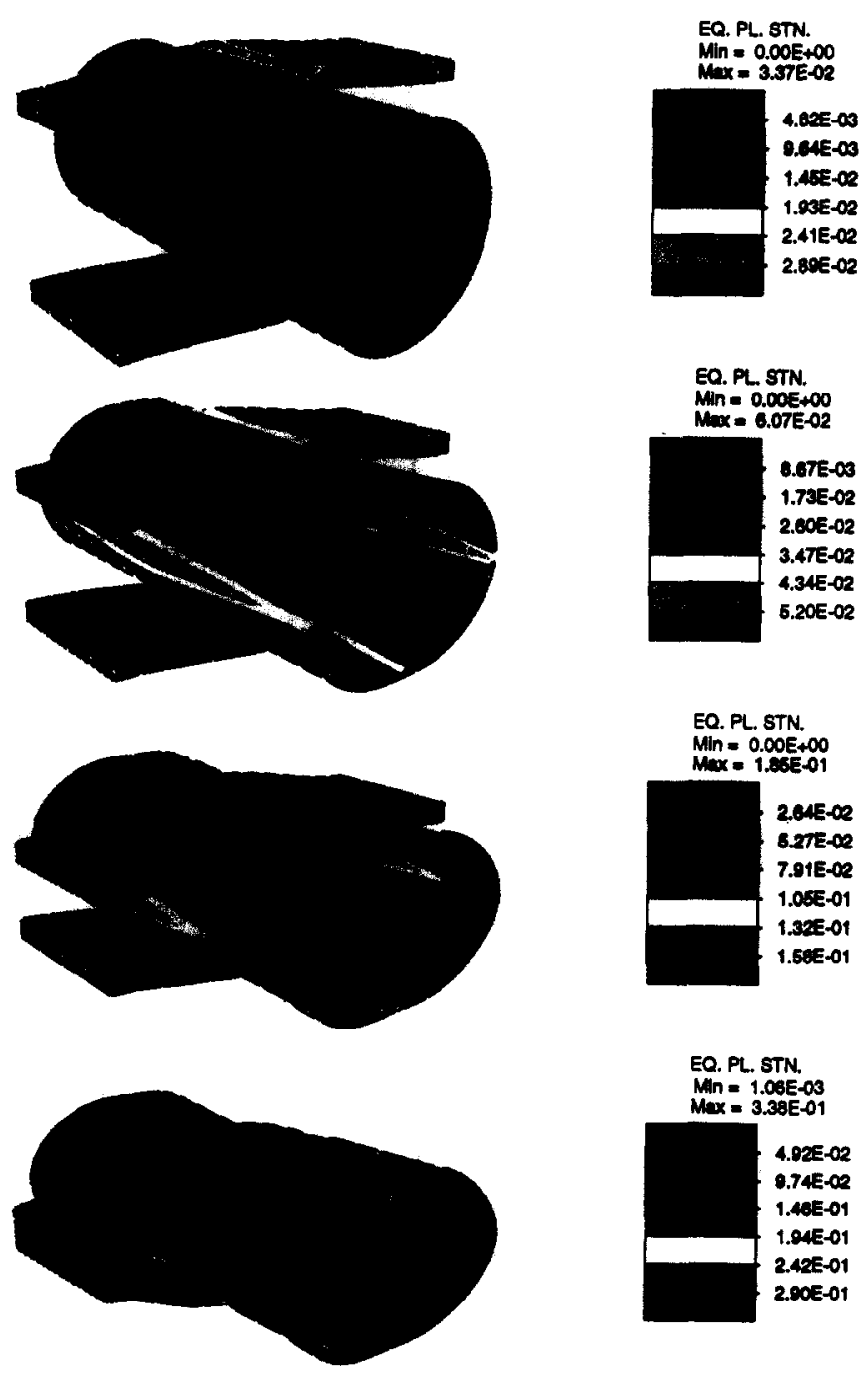

Fig. 7. Pinching of a pipe by two flexible plates. Contour of equivalent plastic strain at a plate edges displacements of $25,50,75$ and $100 \mathrm{~mm}$. 

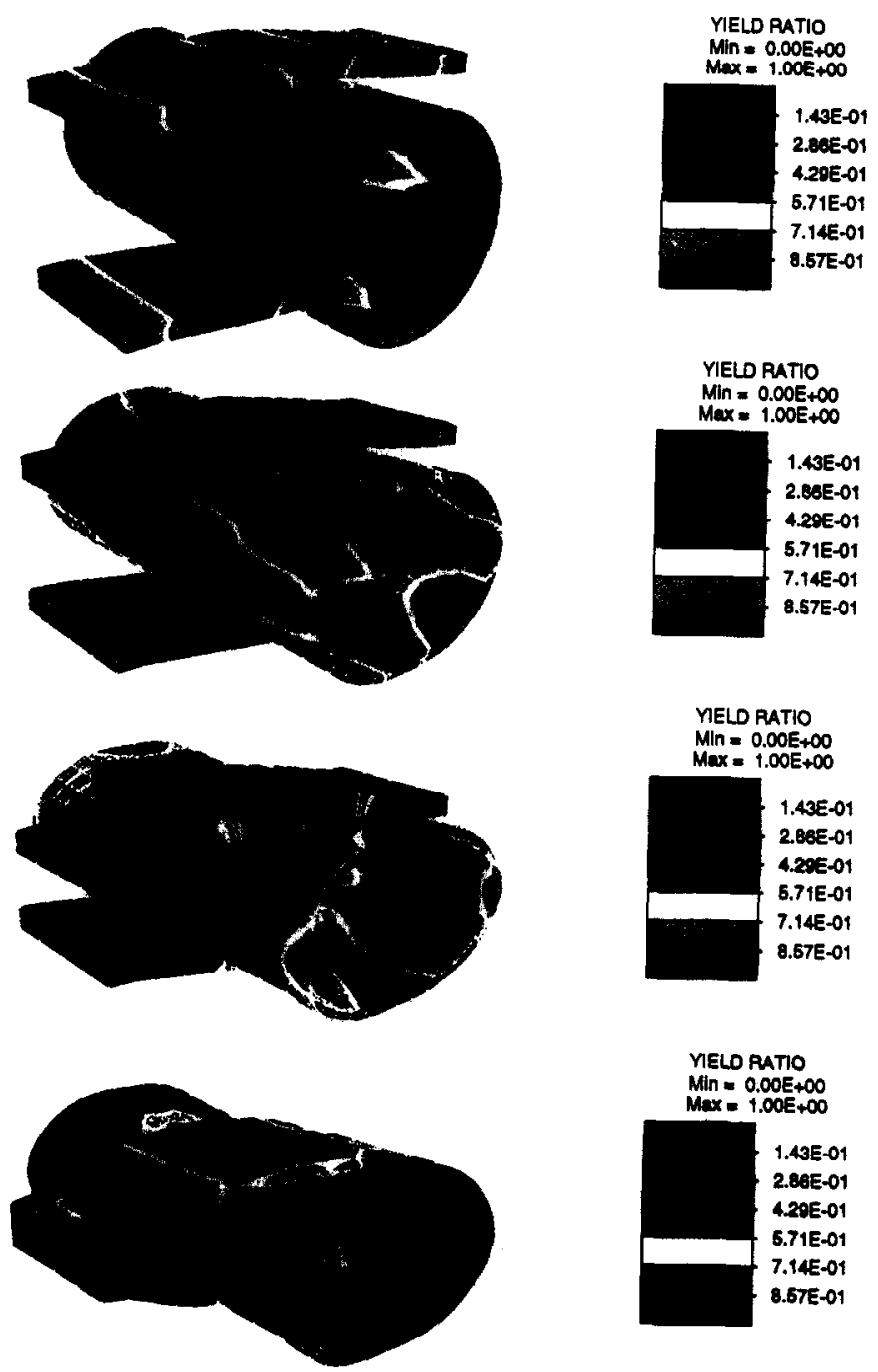

Fig. 8. Pinching of a pipe by two flexible plates. Contour of yield ratio at a plate edges displacements of $25,50,75$ and $100 \mathrm{~mm}$.
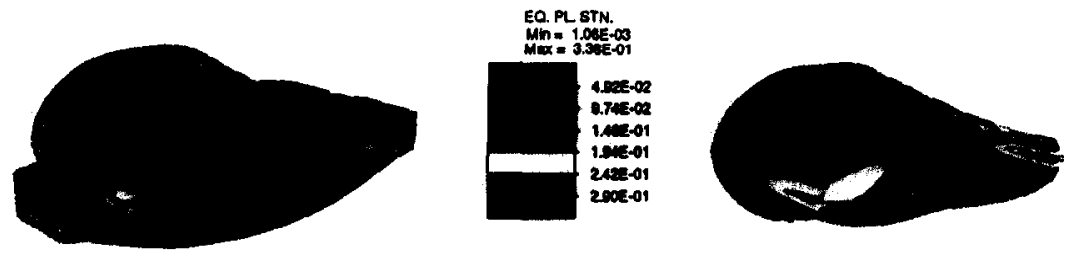

Fig. 9. Pinching of a pipe by two flexible plates. Contour of equivalent plastic strain at the final stage. Half the geometry is represented to appreciate the deformed shape at the middle section of the pipe and the distribution of cquivalent plastic strain at the middle inner part of the pipe.

rollers. The material properties for the strip were taken as bulk modulus $K=171.6 \mathrm{GPa}$, shear modulus $G=79.2 \mathrm{GPa}$ and a hardening law given by the Swift equation

$$
\sigma_{Y}=536.0\left(0.0033+\bar{\varepsilon}^{P}\right)^{0.21} \mathrm{MPa}
$$

The rollers were considered as rigid. The strip was $1 \mathrm{~mm}$ thick and measured $60 \mathrm{~mm}$ length by $2 \mathrm{~mm}$ wide. The rollers were $4 \mathrm{~mm}$ wide and radius of 5 and $2 \mathrm{~mm}$. Relative to the left side of the strip, the center of the rollers were placed at $9,20,31,34$ and $44 \mathrm{~mm}$ as it is indicated in Fig. 10. A Coulomb 


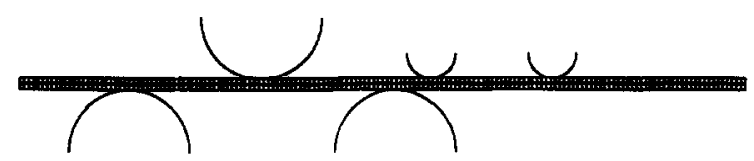

Fig. 10. Draw Bead Simulator. Initial geometry of the strip and initial placement of the rollers.

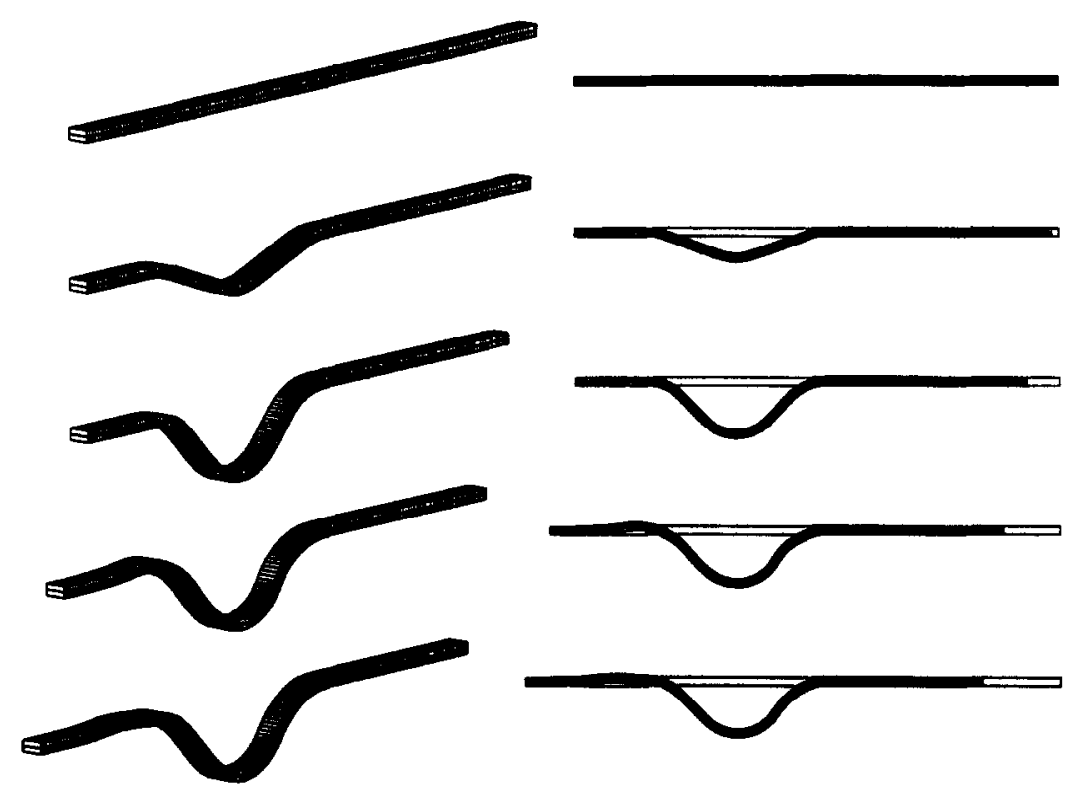

Fig. 11. Draw Bead Simulator. Deformed shapes of the strip at four different stages of the process, corresponding to a displacement of the upper main roller of 3.175 and $6.350 \mathrm{~mm}$ during the first phase and to a prescribed displacement of 3.175 and $6.350 \mathrm{~mm}$ of the left edge of the strip during the second phase, respectively.

friction coefficient of $\mu=0.144$ was assumed between the surfaces of the strip and the rollers. Plane strain conditions have been assumed and only a half part of each roller has been discretized. The loading process consist of two phases. In the first phase, the main upper roller (at the left) is moved downwards to a distance of $6.35 \mathrm{~mm}$ and the left edge of the strip is kept fixed. In the second phase, the rollers are kept fixed and the strip is pulled out from the left to a final distance of $6.35 \mathrm{~mm}$.

The geometry of the problem was modeled with 320 continuum elements being utilized for the discretization of the strip and 100 surface elements being used for the discretization of each of the rollers. A mixed Q1/P0 finite element formulation at finite strains was used for the discretization of the strip. The penalty parameters for contact and friction were taken as $\varepsilon_{\mathrm{N}}=5 \cdot 10^{12}$ and $\varepsilon_{\mathrm{T}}=5 \cdot 10^{10}$. The loading process was achieved in 200 load steps, 100 load steps in each of the phases of the process,

Table 4

Draw bead simulator. Euclidean residual norm for four typical time steps

\begin{tabular}{llll}
\hline Step 50 & Step 100 & Step 150 & Step 200 \\
\hline $5.28310 \mathrm{E}+02$ & $6.53489 \mathrm{E}+02$ & $4.12709 \mathrm{E}+04$ & $4.12687 \mathrm{E}+04$ \\
$3.81569 \mathrm{E}+02$ & $3.58552 \mathrm{E}+02$ & $2.51557 \mathrm{E}+02$ & $1.58693 \mathrm{E}+02$ \\
$2.37672 \mathrm{E}+01$ & $1.14570 \mathrm{E}+02$ & $2.82030 \mathrm{E}+01$ & $3.67684 \mathrm{E}+01$ \\
$1.42762 \mathrm{E}+01$ & $2.48702 \mathrm{E}+01$ & $3.65624 \mathrm{E}+00$ & $6.23131 \mathrm{E}+00$ \\
$3.97753 \mathrm{E}+00$ & $5.95389 \mathrm{E}+00$ & $2.94132 \mathrm{E}-01$ & $2.14350 \mathrm{E}-01$ \\
$1.81214 \mathrm{E}-01$ & $5.91212 \mathrm{E}-01$ & $5.56107 \mathrm{E}-02$ & $9.54870 \mathrm{E}-03$ \\
$5.06938 \mathrm{E}-04$ & $2.36497 \mathrm{E}-03$ & $1.29838 \mathrm{E}-05$ & $2.17244 \mathrm{E}-07$ \\
$5.21445 \mathrm{E}-09$ & $6.42952 \mathrm{E}-08$ & $5.46098 \mathrm{E}-10$ & $5.29836 \mathrm{E}-10$ \\
$5.96470 \mathrm{E}-10$ & $5.03292 \mathrm{E}-10$ & & \\
\hline
\end{tabular}


through displacement control of the main roller and the left edge of the strip. The Newton-Raphson method, combined with a line search optimization procedure, was used to solve the nonlinear system of equations arising from the spatial and temporal discretization. Convergence of the incremental iterative solution procedure was monitored by requiring a tolerance of $10^{-25}$ in the energy norm.
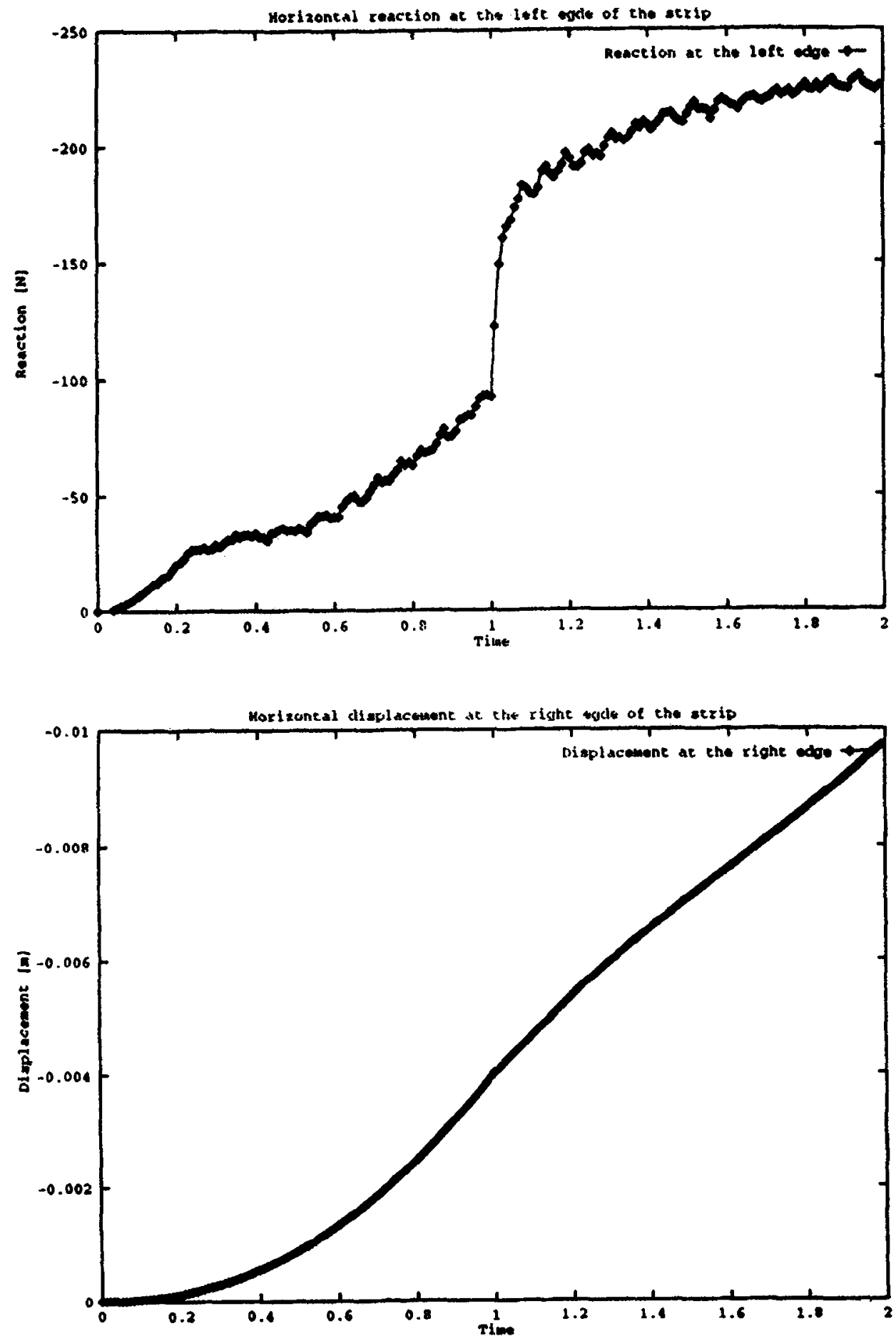

Fig. 12. Draw Bead Simulator. Evolution of the horizontal reaction at the left edge of the strip and evolution of the horizontal displacement of the right edge of the strip. Time scale is such that $t=1$ corresponds to the end of the first phase of the loading process, given by a vertical displacement of the upper main roller of $3.175 \mathrm{~mm}$. 


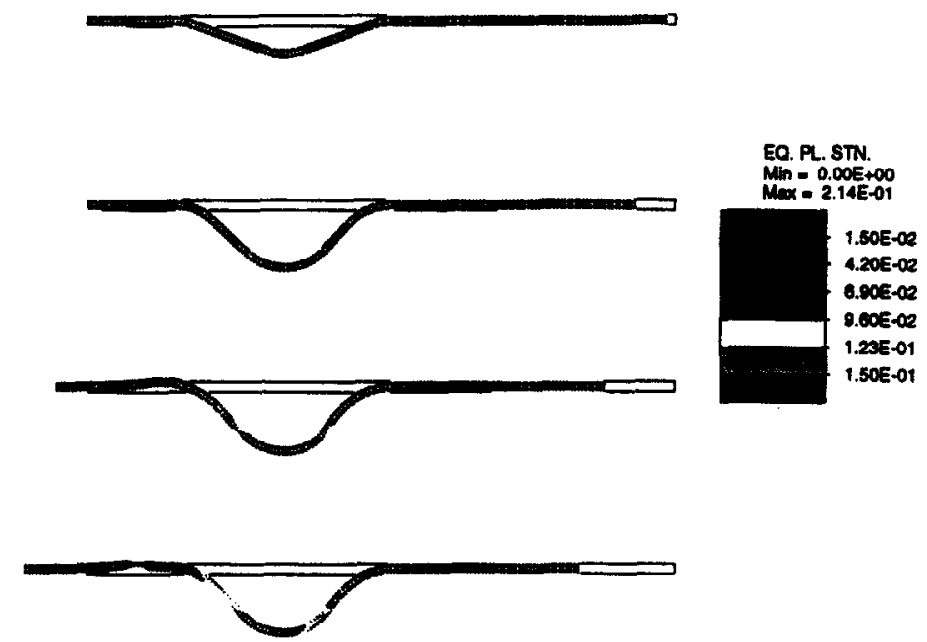

Fig. 13. Draw Bead Simulator. Distribution of the equivalent plastic strain in the strip at four different stages of the process, corresponding to a displacement of the upper main roller of 3.175 and $6.350 \mathrm{~mm}$ during the first phase and to a prescribed displacement of 3.175 and $6.350 \mathrm{~mm}$ of the left edge of the strip during the second phase, respectively.

The analysis was performed in a Silicon Graphics Power Challenge L Workstation and it was accomplished in $2 \mathrm{~h} 5 \mathrm{~min}$ CPU time. Table 4 shows the Euclidean norm of the residual at four typical time steps.

Fig. 10 shows the initial geometry of the strip and the initial placement of the rollers. Fig. 11 shows the deformed shapes of the strip at four different stages of the process, corresponding to a displacement of 3.175 and $6.350 \mathrm{~mm}$ of the upper main roller during the first phase and to prescribed displacements of 3.175 and $6.350 \mathrm{~mm}$ of the left edge of the strip during the second phase, respectively.

Fig. 12 shows the evolution of the horizontal reaction at the left edge of the strip and the evolution of the horizontal displacement of the right edge of the strip, during the loading process. Loading time is such that $t=1$ corresponds to the end of the first phase, with a final vertical displacement of the upper main roller of $6.35 \mathrm{~mm}$.

Fig. 13 shows the distribution of the equivalent plastic strain in the strip at the same four selected stages of the process. A zoom of the central part of the strip with the distribution of equivalent plastic strain at the final stage is shown in Fig. 14.

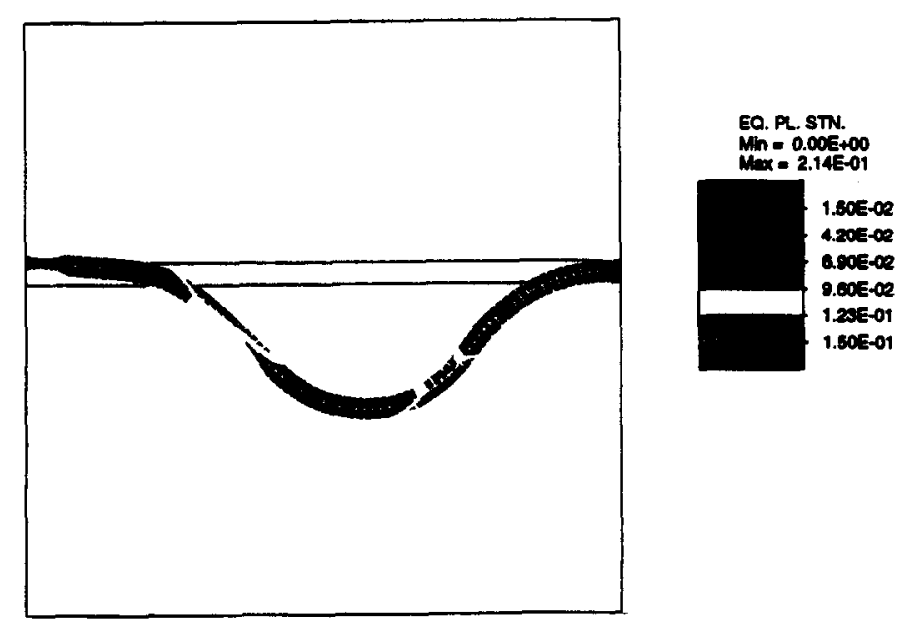

Fig. 14. Draw Bead Simulator. Zoom of the distribution of the equivalent plastic strain in the central part of the strip at the final stage of the process, corresponding to a displacement of the upper main roller of $6.350 \mathrm{~mm}$ during the first phase and to a prescribed displacement of $6.350 \mathrm{~mm}$ of the left edge of the strip during the second phase. 


\section{Concluding remarks}

A new frictional time integration algorithm, suitable for large slip multi-body frictional contact problems at finite deformations has been presented. To introduce the algorithm it has been sufficient to consider a simple model problem: the sliding motion of a particle into a fixed surface. The main aspects of the algorithm can be introduced within the context of this model problem and the extension to a general large multi-body problem at finite deformations can be performed in a straightforward manner.

The key point of the algorithm lies in the parametrization of the incremental assumed slip path as an approximation to a geodesic curve. Remarkably, this parametrization is defined, only in terms of the initial and final position of the particle and the unit normals to the surface at these points, without using the underlying local surface parametrization. This fact allows to deal with large slip situations in a trivial manner.

The algorithm is amenable to exact linearization getting an asymptotic quadratic rate of convergence when used within a Newton-Raphson solution scheme. The performance of the algorithm has been shown in different representative numerical simulations.

\section{Acknowledgment}

Support for his work was provided by the 'Dirección General de Investigación Científica y Técnica, DGICYT' of the 'Ministerio de Educaión y Ciencia, MEC' of Spain, under grant no. 93-013. This support is gratefully acknowledged.

\section{Appendix A. A new frictional time integration algorithm for large slip multi-body frictional contact problems}

In this Appendix a new frictional time integration algorithm, within the framework of finite strains and large slips multi-body frictional contact problems, is introduced.

(A) Notation. Let $2 \leqslant n_{\text {dim }} \leqslant 3$ be the space dimension and $I:=[0, T] \subset \mathbb{R}_{+}$the time interval of interest. Let the open sets $\Omega^{(1)} \subset \mathbb{R}^{n_{\mathrm{dim}}}$ and $\Omega^{(2)} \subset \mathbb{R}^{n_{\mathrm{dim}}}$ with smooth boundaries $\partial \Omega^{(1)}$ and $\partial \Omega^{(2)}$ and closures $\bar{\Omega}^{(1)}:=\Omega^{(1)} \cup \partial \Omega^{(1)}$ and $\bar{\Omega}^{(2)}:=\Omega^{(2)} \cup \partial \Omega^{(2)}$, be the reference placement of two continuum bodies $\mathscr{B}^{(1)}$ and $\mathscr{B}^{(2)}$, with material particles labeled $\boldsymbol{X} \in \bar{\Omega}^{(1)}$ and $Y \in \bar{\Omega}^{(2)}$, respectively.

Denote by $\varphi^{(i)}: \bar{\Omega}^{(i)} \times I \rightarrow \mathbb{R}^{n}$ dim the orientation preserving deformation map of the body $\mathscr{B}^{(i)}$, with material velocities $V^{(i)}:=\partial_{t} \varphi^{(i)}$ and deformation gradients $F^{(i)}:=D \varphi^{(i)}$. For each time $t \in I$, the mapping $t \in I \mapsto \varphi_{t}^{(i)}:=\varphi^{(i)}(\cdot, t)$ represents a one-parameter family of configurations indexed by time $t$, which maps the reference placement of body $\mathscr{B}^{(i)}$ onto its current placement $\mathscr{S}_{t}^{(i)}: \varphi_{t}^{(i)}\left(\mathscr{B}^{(i)}\right) \subset \mathbb{R}^{n_{\mathrm{dim}}}$.

We will denote as the contact surface $\Gamma^{(i)} \subset \partial \Omega^{(i)}$ the part of the boundary of the body $\mathscr{B}^{(i)}$ such that all material points where contact will occur at any time $t \in I$ are included. The current placement of the contact surface $\Gamma^{(i)}$ is given by $\gamma^{(i)}:=\varphi_{t}^{(i)}\left(\Gamma^{(i)}\right)$.

Attention will be focussed to material points on these surfaces denoted as $X \in \Gamma^{(1)}$ and $Y \in \Gamma^{(2)}$. Current placement of these particles is given by $x=\varphi_{i}^{(1)}(X) \in \gamma^{(1)}$ and $y=\varphi_{t}^{(2)}(Y) \in \gamma^{(2)}$.

Using a standard notation in contact mechanics we will assign to each pair of contact surfaces involved in the problem, the roles of slave and master surface. In particular, let $\Gamma^{(1)}$ be the slave surface and $\Gamma^{(2)}$ be the master surface. Additionally, we will denote slave particles and master particles to the material points of the slave and master surfaces, respectively. With this notation in hand, we will require that any slave particle may not penetrate the master surface, at any time $t \in I$. Although in the continuum setting the slave-master notation plays no role, in the discrete setting this choice becomes important.

(B) Parametrization of contact surfaces. Let $\mathscr{A}^{(i)} \subset \mathbb{R}^{n} \mathrm{dim}^{-1}$ be a parent domain for the contact surface of body $\mathscr{B}^{(i)}$. A parametrization of the contact surface for each body $\mathscr{B}^{(i)}$ is introduced by a family of (orientation preserving) one-parameter mappings indexed by time, $\psi_{t}^{(i)}: \mathscr{A}^{(i)} \subset$ 
$\mathbb{R}^{n} \operatorname{dim}^{-1} \rightarrow \mathbb{R}^{n} \mathrm{dim}$ such that $\Gamma^{(i)}:=\boldsymbol{\psi}_{0}^{(i)}\left(\mathscr{A}^{(i)}\right)$ and $\gamma^{(i)}:=\boldsymbol{\psi}_{t}^{(i)}\left(\mathscr{A}^{(i)}\right)$. Using the mapping composition rule, it also follows that $\boldsymbol{\psi}_{t}^{(i)}=\boldsymbol{\varphi}_{t}^{(i)} \circ \boldsymbol{\psi}_{0}^{(i)}$. In what follows, it will be assumed that these parametrizations have the required smoothness conditions. Additionally, we will consider the case $n_{\mathrm{dim}}=3$. Particularization for $n_{\text {dim }}=2$ is trivial after the three-dimensional case has been considered.

Within the slave-master surface role focus will be placed on the parametrization of the master surface. Using the parametrization of the contact surfaces introduced above we consider a point $\xi:=\left(\xi^{1}, \xi^{2}\right) \in \mathscr{A}^{(2)}$ of the parent domain, such that

$$
\boldsymbol{Y}:-\boldsymbol{\psi}_{0}^{(2)}(\boldsymbol{\xi}), \quad \boldsymbol{y}:=\boldsymbol{\psi}_{t}^{(2)}(\boldsymbol{\xi})
$$

Attached to each master particle $\boldsymbol{Y} \in \Gamma^{(2)}$ we introduce the convected surface basis $\boldsymbol{E}_{\alpha}(\boldsymbol{\xi})$ and $\boldsymbol{e}_{\alpha}(\boldsymbol{\xi})$, $\alpha=1,2$ on the reference and current configurations, respectively, as

$$
\boldsymbol{E}_{\alpha}(\xi):=\boldsymbol{\psi}_{0, \alpha}^{(2)}(\xi), \quad \boldsymbol{e}_{\alpha}(\xi):=\boldsymbol{\psi}_{i, \alpha}^{(2)}(\xi)
$$

where $(\cdot)_{, \alpha}$ denotes partial derivative with respect to $\xi^{\alpha}$.

(C) Contact normal constraints. Let $\bar{y}(X, t) \in \gamma^{(2)}$ be the closest-point projection of the current position of the slave particle $\boldsymbol{X}$ onto the current placement of the master surface $\Gamma^{(2)}$, defined as

$$
\begin{aligned}
& \overline{\boldsymbol{Y}}(\boldsymbol{X}, t):=\arg \min _{\boldsymbol{Y} \in \Gamma^{(2)}}\left\{\left\|\boldsymbol{\varphi}_{t}^{(1)}(\boldsymbol{X})-\boldsymbol{\varphi}_{t}^{(2)}(\boldsymbol{Y})\right\|\right\} \\
& \overline{\boldsymbol{y}}(\boldsymbol{X}, t):=\boldsymbol{\varphi}_{t}^{(2)}(\overline{\boldsymbol{Y}})
\end{aligned}
$$

and let $g_{\mathrm{N}}(X, t)$ be the gap function defined for any slave particle $X \in \Gamma^{(1)}$ and for any time $t \in I$ as

$$
g_{\mathrm{N}}(X, t):=-\left[\boldsymbol{\varphi}_{t}^{(1)}(X)-\varphi_{t}^{(2)}(\bar{Y}(X, t))\right] \cdot \nu
$$

where $\nu: y^{(2)} \rightarrow S^{2}$ is the unit outward normal field to the current placement of the master surface particularized at the closest-point projection $\bar{y}(X, t) \in \gamma^{(2)}$.

Let $P^{(1)}(X, t)$ be the first Piola-Kirchhoff stress tensor and $N^{(1)}(X)$ the unit outward normal to the slave surface $\Gamma^{(1)}$ in the reference configuration. The nominal (Piola) frictional contact traction at $\boldsymbol{X} \in \Gamma^{(1)}$ is given as

$$
\boldsymbol{t}^{(1)}(X, t)=\boldsymbol{P}^{(1)}(X, t) \cdot \boldsymbol{N}^{(1)}(X)
$$

and the contact nominal pressure $t_{\mathrm{N}}(X, t)$ is defined as

$$
t_{\mathrm{N}}(X, t):=t^{(1)}(X, t) \cdot \nu
$$

With the preceding notation in hand, the contact normal constraints of impenetrability, non-adhesion and contact persistency can be written in Kuhn-Tucker form as

$$
\begin{aligned}
& t_{\mathrm{N}} \geqslant 0, \quad g_{\mathrm{N}} \leqslant 0, \quad t_{\mathrm{N}} g_{\mathrm{N}}=0 \\
& t_{\mathrm{N}} \dot{g}_{\mathrm{N}}=0 \quad \text { if } t_{\mathrm{N}}>0
\end{aligned}
$$

(D) Convected basis, metric and curvature tensors at the closest-point projection. Associated to the clusest-point projection given by (A.3)-(A.4), for some point $\bar{\xi}:=\left(\bar{\xi}^{1}, \bar{\xi}^{2}\right) \in \mathscr{A}^{(2)}$ of the parent domain we will have

$$
\overline{\boldsymbol{Y}}(X, t):=\boldsymbol{\psi}_{0}^{(2)}(\bar{\xi}(X, t)), \quad \bar{y}(X, t):=\boldsymbol{\psi}_{t}^{(2)}(\bar{\xi}(X, t))
$$

Attached to the master particle $\bar{Y}(X, t) \in \Gamma^{(2)}$ we define the convected surface basis on the reference and current configurations, respectively, as

$$
\tau_{\alpha}^{\mathrm{ref}}(X, t):=E_{\alpha}(\bar{\xi}(X, t)), \quad \tau_{\alpha}(X, t):=e_{\alpha}(\bar{\xi}(X, t))
$$

Additionally, the unit outward normals $\nu^{\text {ref }} \in S^{2}$ and $\nu \in S^{2}$ at the master particle $\overline{\boldsymbol{Y}}(\boldsymbol{X}, t)$ on the reference and current configurations, respectively, can be defined as 


$$
\nu^{\mathrm{ref}}:=\frac{\tau_{1}^{\mathrm{ref}} \times \tau_{2}^{\mathrm{ref}}}{\left\|\tau_{1}^{\mathrm{ref}} \times \tau_{2}^{\mathrm{ref}}\right\|}, \quad \nu:=\frac{\tau_{1} \times \tau_{2}}{\left\|\tau_{1} \times \tau_{2}\right\|}
$$

The vectors $\tau_{\alpha}^{\text {ref }} \in T_{\nu \text { ref }} S^{2}$ and $\tau_{\alpha} \in T_{\nu} S^{2}, \alpha=1,2$ span the tangent spaces $T_{\nu}$ ref $S^{2}$ and $T_{\nu} S^{2}$ to the $S^{2}$ unit sphere at $\nu^{\text {ref }}$ and $\boldsymbol{\nu}$, respectively. Here, the tangent space to the $S^{2}$ unit sphere at $\nu \in S^{2}$ is defined as

$$
T_{\nu} S^{2}:=\left\{\delta \boldsymbol{\nu} \in \mathbb{R}^{n_{\mathrm{dim}}}: \delta \boldsymbol{\nu} \cdot \boldsymbol{\nu}=0\right\}
$$

The convected surface basis vectors $\tau_{\alpha}^{\text {ref }}$ and $\tau_{\alpha}, \alpha=1,2$, augmented with the unit outward normals $\nu^{\text {ref }}$ and $\nu$, provides local spatial frames at the master particle $\bar{Y}(X, t)$ on the reference and current configurations, respectively.

The convected surface basis vectors $\tau_{\alpha}^{\text {ref }}$ and $\tau_{\alpha}, \alpha=1,2$, induces a surface metric or first fundamental form on the reference and current configurations, defined respectively as

$$
M_{\alpha \beta}:=\tau_{\alpha}^{\mathrm{ref}} \cdot \tau_{\beta}^{\mathrm{ref}}, \quad m_{\alpha \beta}:=\tau_{\alpha} \cdot \tau_{\beta}
$$

Inverse surface metrics $M^{\alpha \beta}$ and $m^{\alpha \beta}$ are defined in the usual manner. Additionally, dual surface basis on the surface and current configurations are straightforward defined respectively as

$$
\tau_{\mathrm{ref}}^{\alpha}:=M^{\alpha \beta} \tau_{\beta}^{\mathrm{ref}}, \quad \tau^{\alpha}:=m^{\alpha \beta} \tau_{\beta}
$$

The variation of the convected surface basis along the convected coordinates, together with the unit normal, induces the second fundamental form or surface curvature defined, on the reference and current configurations, as

$$
\kappa_{\alpha \beta}^{\text {ref }}:=E_{\alpha, \beta}(\bar{\xi}) \cdot \nu^{\text {ref }}, \quad \kappa_{\alpha \beta}:=e_{\alpha, \beta}(\bar{\xi}) \cdot \nu
$$

(E) Constrained frictional evolution problem. The relative slip velocity in the convected configuration is defined as

$$
\boldsymbol{v}_{\mathrm{T}}^{\mathrm{ref}}(\boldsymbol{X}, t):=\dot{\boldsymbol{Y}}(\boldsymbol{X}, t)
$$

The relative slip velocity in the current configuration $v_{\mathrm{T}}$ is defined as the push-forward of the convected slip velocity and takes the form

$$
\boldsymbol{v}_{\mathrm{T}}(\boldsymbol{X}, t):=\boldsymbol{F}_{t}^{(2)}(\bar{\xi}(\boldsymbol{X}, t)) \cdot \boldsymbol{v}_{\mathrm{T}}^{\mathrm{ref}}(\boldsymbol{X}, t)
$$

We define the nominal frictional tangent traction $t_{\mathrm{T}}(X, t)$ as (minus) the projection of the nominal frictional contact traction $t^{(1)}(X, t)$ onto the unit normal $\nu$, as

$$
t_{\mathrm{T}}(\boldsymbol{X}, t):=-\mathbb{P}_{\nu} t^{(1)}(\boldsymbol{X}, t)=t_{\mathrm{T}}^{\alpha}(\boldsymbol{X}, t) \tau_{\alpha}
$$

With the preceding notation in hand the slip rule and frictional constraints can be written in Kuhn-Tucker form as

$$
\begin{aligned}
& v_{\mathrm{T}}^{b}(X, t)=\gamma p_{\mathrm{T}}^{b} \\
& \Phi\left(t_{\mathrm{T}}^{\mathrm{b}}, t_{\mathrm{N}}\right) \leqslant 0, \quad \gamma \geqslant 0, \quad \gamma \Phi\left(t_{\mathrm{T}}^{\mathrm{b}}, t_{\mathrm{N}}\right)=0 \\
& \gamma \dot{\Phi}\left(t_{\mathrm{T}}^{\mathrm{b}}, t_{\mathrm{N}}\right)=0 \quad \text { if } \Phi\left(t_{\mathrm{T}}^{\mathrm{b}}, t_{\mathrm{N}}\right)=0
\end{aligned}
$$

where $\Phi\left(t_{\mathrm{T}}^{\mathrm{b}}, t_{\mathrm{N}}\right)$ is the slip function defining the admissible nominal traction states, $\boldsymbol{p}_{\mathrm{T}}^{\mathrm{b}}$ is' the gradient of the slip potential, usually defined as the slip function for constant pressure, $\gamma$ is the slip rate and $(\cdot)^{b}$ states for the one-form associated to the corresponding vector object.

(F) Regularized problem. The penalty regularization of (A.8) and (A.19) defines the following regularized constrained frictional evolution problem. The regularized normal constraints are defined as

$$
t_{\mathrm{N}}(X, t):=\epsilon_{\mathrm{N}}\left\langle g_{\mathrm{N}}(X, t)\right\rangle
$$


where $\langle\cdot\rangle$ is the Macauley bracket, representing the positive part of its operand. The regularized constrained frictional evolution equations are defined as

$$
\boldsymbol{v}_{\mathrm{T}}^{\mathrm{b}}(\boldsymbol{X}, t)=\gamma \boldsymbol{p}_{\mathrm{T}}^{\mathrm{b}}+\frac{1}{\epsilon_{\mathrm{T}}} \mathscr{L}_{v_{\mathrm{T}}} \boldsymbol{t}_{\mathrm{T}}^{\mathrm{b}}
$$

subjected to the following constraints

$$
\begin{aligned}
& \Phi\left(t_{\mathrm{T}}^{b}, t_{\mathrm{N}}\right) \leqslant 0, \quad \gamma \geqslant 0, \quad \gamma \Phi\left(t_{\mathrm{T}}^{b}, t_{\mathrm{N}}\right)=0 \\
& \gamma \dot{\Phi}\left(t_{\mathrm{T}}^{\mathrm{b}}, t_{\mathrm{N}}\right)=0 \quad \text { if } \Phi\left(t_{\mathrm{T}}^{\mathrm{b}}, t_{\mathrm{N}}\right)=0
\end{aligned}
$$

where $\mathscr{L}_{v_{T}} t_{\mathrm{T}}$ is the Lie derivative of the frictional tangent traction along the flow induced by the relative slip velocity $\boldsymbol{v}_{\mathrm{T}}$, defined as

$$
\mathscr{L}_{v_{\mathrm{T}}} t_{\mathrm{T}}^{b}:=\dot{t}_{\mathrm{T}_{\alpha}} \tau^{\alpha}
$$

Within the context of the product formula algorithms, a frictional operator split of the constrained evolution problem can be introduced by means of a trial state, defined by freezing the irreversible (plastic) slip response, i.e. setting $\gamma=0$, as follows:

$$
\begin{array}{lll}
\text { Problem 1: Trial state } & \text { Problem 2: Return mapping } \\
\left\{\begin{array}{l}
\mathscr{L}_{v_{\mathrm{T}}} t_{\mathrm{T}}^{\mathrm{b}}:=\epsilon_{\mathrm{T}} \boldsymbol{v}_{\mathrm{T}}^{\mathrm{b}} \\
\text { unconstrained }
\end{array}\right\} & \left\{\begin{array}{l}
\mathscr{L}_{v_{\mathrm{T}}} t_{\mathrm{T}}^{\mathrm{b}}:=-\epsilon_{\mathrm{T}} \gamma \partial_{t_{\mathrm{T}}^{\mathrm{b}}} \Phi\left(t_{\mathrm{T}}^{\mathrm{b}}, t_{\mathrm{N}}, \alpha\right) \\
\Phi\left(t_{\mathrm{T}}^{\mathrm{b}}, t_{\mathrm{N}}\right) \leqslant 0, \quad \gamma \geqslant 0, \quad \gamma \Phi\left(t_{\mathrm{T}}^{\mathrm{b}}, t_{\mathrm{N}}\right)=0
\end{array}\right\}
\end{array}
$$

(G) Frictional time integration algorithm. A step-by-step description of the new frictional time integration algorithm, suitable for large slips at finite deformations, is as follows:

Step 1. Initial conditions: Database. Consider given as initial conditions at time $t_{n}$, the nominal frictional traction on reference configuration $\boldsymbol{t}_{\mathrm{T}_{n}}^{\text {ref }}$, the isoparametric coordinates $\bar{\xi}_{n}$ of the closest-point projection at the last converged time step and the master element surface $\Gamma_{n}^{e}$ containing this point. Using the maps, $\psi_{0}^{(i)}: \mathscr{A}^{(i)} \subset \mathbb{R}^{n_{\mathrm{dim}}{ }^{-1}} \rightarrow \Gamma^{(2)} \subset \mathbb{R}^{n_{\mathrm{dim}}}$ and $\boldsymbol{\psi}_{t}^{(i)}: \mathscr{A}^{(i)} \subset \mathbb{R}^{n_{\mathrm{dim}}{ }^{-1}} \rightarrow \gamma^{(2)} \subset \mathbb{R}^{n_{\mathrm{dim}}}$ introduced above, the reference and current placement of the closest-point projection at time $t_{n}$ can be written as $\overline{\boldsymbol{Y}}_{n}:=\psi_{0}^{(2)}\left(\overline{\boldsymbol{\xi}}_{n}\right)$ and $\bar{y}_{n}:=\psi_{n}^{(2)}\left(\overline{\boldsymbol{\xi}}_{n}\right)$, respectively.

Within the deformation-driven framework, consider a prescribed deformation leading at time $t_{n+1}$ to a prescribed value of the isoparametric coordinates of the current clusest-point projection $\bar{\xi}_{n+1}$ onto the master element surface $\Gamma_{n+1}^{e}$ containing this point. Reference and current placements of this point can be written as $\overline{\boldsymbol{Y}}_{n+1}:=\psi_{0}^{(2)}\left(\overline{\boldsymbol{\xi}}_{n+1}\right)$ and $\overline{\boldsymbol{y}}_{n+1}:=\psi_{n+1}^{(2)}\left(\overline{\boldsymbol{\xi}}_{n+1}\right)$, respectively.

Step 2. Definition of the local orthonormal frames at $\bar{\xi}_{n}$ and $\bar{\xi}_{n+1}$ on reference configuration. As it was shown in Section 2 we define two sets of local orthonormal frames at the initial $\overline{\boldsymbol{\xi}}_{n}$ and final $\overline{\boldsymbol{\xi}}_{n+1}$ points on reference configuration. We will denote the basis vectors defining the local frames as $\left\{\boldsymbol{\nu}_{n}^{\text {ref }}, \hat{\boldsymbol{\tau}}_{\alpha_{n}}^{\text {ref }}\right\}$ and $\left\{\boldsymbol{\nu}_{n+1}^{\text {ref }}, \hat{\boldsymbol{\tau}}_{\alpha_{n+1}}^{\text {ref }}\right\}$, with $\alpha=1,2$. Here, $\boldsymbol{\nu}_{n}^{\text {ref }} \in S^{2}$ and $\boldsymbol{\nu}_{n+1}^{\text {ref }} \in S^{2}$ are the unit outward normals to the master surface elements $\Gamma_{n}^{e}$ and $\Gamma_{n+1}^{e}$ at the points $\overline{\boldsymbol{\xi}}_{n}$ and $\bar{\xi}_{n+1}$, respectively, on the reference configuration. The unit tangent vectors $\hat{\boldsymbol{\tau}}_{1_{n}}^{n+f} \in T_{\nu \text { ref }} S^{2}$ and $\hat{\boldsymbol{\tau}}_{1_{n+1}}^{\text {ref }} \in T_{\nu \text { ref }} S^{2}$ are defined as it was shown in Section 2, in such a way that they are tangent to the assumed slip path. We would like to remark that restrictions of the type $\Gamma_{n}^{e} \equiv \Gamma_{n+1}^{e}$ or $\bar{\Gamma}_{n}^{e} \cap \bar{\Gamma}_{n+1}^{e} \neq \emptyset$ are not necessary to be considered in this framework.

Step 3. Trial frictional traction on reference configuration. The trial frictional traction on reference configuration can be computed in a two-step algorithm:

(i) Parallel transport of the frictional traction $t_{\mathrm{T}_{n}}^{b^{\text {ret }}}$ on reference configuration at time $t_{n}$, along the slip path from $\overline{\boldsymbol{\xi}}_{n}$ to $\overline{\boldsymbol{\xi}}_{n+1}$ on reference configuration.

(ii) Trial slip contribution, using a $\mathrm{BE}$ algorithm.

This two-step algorithm leads to the following expression

$$
\boldsymbol{t}_{\mathrm{T}_{n+1}}^{b^{\text {ref trial }}}:=\overline{\boldsymbol{A}} \cdot \boldsymbol{t}_{\mathrm{T}_{n}}^{\text {ref }}+\boldsymbol{\epsilon}_{\mathrm{T}} \Gamma^{\text {slip }} \hat{\boldsymbol{\tau}}_{i_{n+1}}^{\text {ref }}
$$


Table A.1

Frictional time integration algorithm at finite deformations

1. Initial conditions at time $t_{n}$ : Database. Given $\left\{t_{\mathrm{r}_{n}}^{\text {ref }}, \overline{\boldsymbol{\xi}}_{n}, \boldsymbol{\nu}_{n}^{\text {ref }}\right\}$ for the (one form) frictional traction, isoparametric coordinates and unit outward normal in reference configuration (at the slave quadrature points) and for prescribed (motion driven) values $\left\{\bar{\xi}_{n+1}, \nu_{n+1}\right\}$

Set:

$$
\begin{array}{ll}
\overline{\boldsymbol{Y}}_{n}:=\psi_{0}^{(2)}\left(\overline{\boldsymbol{\xi}}_{n}\right), & \overline{\boldsymbol{Y}}_{n+1}:=\psi_{0}^{(2)}\left(\overline{\boldsymbol{\xi}}_{n+1}\right) \\
\overline{\boldsymbol{y}}_{n}:=\psi_{n}^{(2)}\left(\overline{\boldsymbol{\xi}}_{n}\right), & \overline{\boldsymbol{y}}_{n+1}:=\psi_{n+1}^{(2)}\left(\overline{\boldsymbol{\xi}}_{n+1}\right)
\end{array}
$$

2. Definition of local orthonormal frames attached to $\mathbf{Y}_{n}$ and $\mathbf{Y}_{n+1}$ induced by the slip path.

Set

$$
\begin{aligned}
& \hat{\boldsymbol{\tau}}_{2_{n}}^{\text {ref }}:=\frac{\boldsymbol{\nu}_{n}^{\text {ref }} \times \boldsymbol{m}}{\left\|\boldsymbol{\nu}_{n}^{\text {ref }} \times \boldsymbol{m}\right\|}, \quad \hat{\boldsymbol{\tau}}_{1_{n}}^{\text {ref }}:=\hat{\boldsymbol{\tau}}_{2_{n}}^{\text {ref }} \times \boldsymbol{\nu}_{n}^{\text {ref }} \\
& \hat{\boldsymbol{\tau}}_{2_{n+1}}^{\text {ref }}:=\frac{\boldsymbol{\nu}_{n+1}^{\text {ref }} \times \boldsymbol{m}}{\left\|\boldsymbol{\nu}_{n+1}^{\text {ref }} \times \boldsymbol{m}\right\|}, \quad \hat{\boldsymbol{\tau}}_{1_{n+1}}^{\text {ref }}:=\hat{\boldsymbol{\tau}}_{2_{n+1}}^{\text {ref }} \times \boldsymbol{\nu}_{n+1}^{\text {ref }} \quad \text { where } \\
& \boldsymbol{d}=\overline{\boldsymbol{Y}}_{n+1}-\overline{\boldsymbol{Y}}_{n}, \quad \boldsymbol{d}=\|\boldsymbol{d}\|, \quad \boldsymbol{m}=\boldsymbol{d} / \boldsymbol{d}
\end{aligned}
$$

3. Slip path parametrization and slip amount.

(i) Slip path parametrization

$$
\zeta \in[-1,+1] \mapsto \bar{Y}(\zeta):=\mathscr{H}^{1}(\zeta) \bar{Y}_{n}+\frac{d}{2 \cos \Theta_{n}} \overline{\mathscr{H}}^{1}(\zeta) \hat{\boldsymbol{\tau}}_{1_{n}}^{\mathrm{ref}}+\mathscr{H}^{2}(\zeta) \overline{\boldsymbol{Y}}_{n+1}+\frac{d}{2 \cos \Theta_{n+1}} \overline{\mathscr{H}}^{2}(\zeta) \hat{\tau}_{1_{n+1}}^{\mathrm{ref}}
$$

where

$$
\begin{array}{ll}
\cos \Theta_{n}:=\hat{\tau}_{1_{n}}^{\text {ref }} \cdot \boldsymbol{m}, \quad \cos \Theta_{n+1}:=\hat{\boldsymbol{\tau}}_{1_{n+1}}^{\text {ref }} \cdot \boldsymbol{m} \\
\mathscr{H}^{1}(\zeta)=(2+\zeta)(1-\zeta)^{2} / 4, \quad \overline{\mathscr{H}}^{1}(\zeta)=(1+\zeta)(1-\zeta)^{2} / 4 \\
\mathscr{H}^{2}(\zeta)=(2-\zeta)(1+\zeta)^{2} / 4, \quad \overline{\mathscr{H}}^{2}(\zeta)=-(1-\zeta)(1+\zeta)^{2} / 4
\end{array}
$$

(ii) Tangent map

$$
\zeta \in[-1,+1] \mapsto \overline{\boldsymbol{Y}}_{\mathrm{T}}(\zeta):=\nabla \mathscr{H}^{1}(\zeta) \overline{\boldsymbol{Y}}_{n}+\frac{d}{2 \cos \Theta_{n}} \nabla \overline{\mathscr{K}}^{1}(\zeta) \hat{\tau}_{1_{n}}^{\mathrm{ref}}+\nabla \mathscr{K}^{2}(\zeta) \overline{\boldsymbol{Y}}_{n+1}+\frac{d}{2 \cos \Theta_{n+1}} \nabla \overline{\mathscr{K}}^{2}(\zeta) \hat{\tau}_{1_{n+1}^{\mathrm{ref}}}
$$

(iii) Slip amount

$$
\Gamma^{\mathrm{slip}}=\sum_{l=1}^{N_{\mathrm{int}}} W_{I}\left\|\overline{\boldsymbol{Y}}_{\mathrm{T}}\left(\zeta_{I}\right)\right\|
$$

4. Trial state.

(i) Trial frictional traction on reference configuration

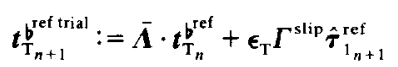

where

$$
\vec{\Lambda}:=\hat{\boldsymbol{\tau}}_{\boldsymbol{\alpha}_{n+1}}^{\mathrm{ref}} \otimes \hat{\boldsymbol{\tau}}_{\boldsymbol{\alpha}_{n}}^{\mathrm{ref}}, \quad \alpha=1,2
$$

(ii) Push-forward to the current configuration

$$
t_{\mathrm{T}_{n+1}}^{\text {trial }}:=\varphi_{*}^{(2)}\left(\boldsymbol{t}_{\mathrm{T}_{n+1}}^{\mathrm{b}_{\text {ref trial }}^{\text {tis }}}\right)=\left(\tau_{n+1}^{\alpha} \otimes \tau_{\alpha_{n+1}}^{\text {ref }}\right) \cdot t_{\mathrm{T}_{n+1}}^{\text {bcf trial }}, \quad \alpha=1,2
$$

(iii) Trial slip function

$$
\Phi_{n+1}^{\text {trial }}:=\left\|t_{\mathrm{r}_{n+1}}^{b_{\text {trial }}}\right\|-\mu t_{\mathrm{N}_{n+1}}
$$

5. Frictional return mapping: Projection onto the Coulomb disk.

(i) Return mapping

$$
t_{\mathrm{T}_{n+1_{\alpha}}}= \begin{cases}t_{\mathrm{T}_{n+1}}^{\text {trial }} & \text { if } \Phi_{n+1}^{\text {trial }} \leqslant 0 \text { (stick) } \\ \mu t_{\mathrm{N}_{n+1}} p_{\mathrm{T}_{n+1}}^{\text {trial }} & \text { otherwise (slip) }\end{cases}
$$

where

$$
\boldsymbol{p}_{\mathrm{T}_{n+1}}^{b_{\text {trial }}}=\boldsymbol{t}_{\mathrm{T}_{n+1}}^{t_{\text {irial }}} /\left\|t_{\mathrm{T}_{n+1}}^{t_{\text {rial }}}\right\|
$$


where $\Gamma_{\text {slip }}$ is the slip amount on the reference configuration computed as it was shown in Section 2 and $\bar{\Lambda} \in \mathrm{SO}(3)$ is the shifter or orthogonal parallel transport operator defined as

$$
\overline{\boldsymbol{\Lambda}}:=\hat{\boldsymbol{\tau}}_{\alpha_{n+1}}^{\mathrm{ref}} \otimes \hat{\boldsymbol{\tau}}_{\alpha_{n}}^{\mathrm{ref}}, \quad \boldsymbol{\alpha}=1,2
$$

where $\mathrm{SO}(3)$ is the special orthogonal group defined as

$$
\operatorname{SO}(3):=\left\{\boldsymbol{\Lambda}: \mathbb{R}^{3} \rightarrow \mathbb{R}^{3} \mid \boldsymbol{\Lambda}^{\mathrm{T}}=\boldsymbol{\Lambda}^{-1} \text { and } \operatorname{det}[\boldsymbol{\Lambda}]=+1\right\}
$$

Step 4. Push-forward to the current configuration. Once the (one-form) nominal frictional traction at $\overline{\boldsymbol{\xi}}_{n+1}$ on reference configuration has been computed, we perform the push-forward to the current configuration, defined as

$$
\boldsymbol{t}_{\mathrm{T}_{n+1}}^{b_{\text {trial }}}:=\boldsymbol{\varphi}_{*}^{(2)}\left(\boldsymbol{t}_{\mathrm{T}_{n+1}}^{\text {ref trial }}\right)=\left(\boldsymbol{\tau}_{n+1}^{\alpha} \otimes \boldsymbol{\tau}_{\alpha_{n+1}}^{\text {ref }}\right) \cdot \boldsymbol{t}_{\mathbf{T}_{n+1}}^{b_{\text {ref trial }}}, \quad \alpha=1,2
$$

where $\boldsymbol{\tau}_{n+1}^{\alpha}$ and $\boldsymbol{\tau}_{\alpha_{n+1}}^{\text {ref }}, \alpha=1,2$ are the reciprocal convective basis at $\overline{\boldsymbol{\xi}}_{n+1}$ on current configuration and the natural convective basis at $\overline{\boldsymbol{\xi}}_{n+1}$ on reference configuration, respectively.

Step 5. Projection onto the Coulomb disk. Return mapping algorithm. The computed trial frictional traction on current configuration is projected onto the Coulomb disk via a return mapping algorithm. This projection, involves the computation of the trial slip function defined as

$$
\Phi_{n+1}^{\text {trial }}:=\left\|t_{\mathrm{T}_{n+1}}^{\text {trial }}\right\|-\mu t_{\mathrm{N}_{n+1}}
$$

If $\Phi_{n+1}^{\text {trial }} \leqslant 0$ then the trial frictional traction satisfies the frictional constraints and the projection is just

$$
\boldsymbol{t}_{\mathrm{T}_{n+1}}^{\mathrm{b}}:=\boldsymbol{t}_{\mathrm{T}_{n+1}}^{\mathrm{b}_{\mathrm{trial}}}
$$

otherwise, a projection of the trial values onto the Coulomb disk must be done. For the simple classical Coulomb model, the return mapping algorithm leads to a direct computation of the frictional tangent traction given by

$$
\boldsymbol{t}_{\mathrm{T}_{n+1}}^{\mathrm{b}}:=\mu t_{\mathrm{N}_{n+1}} p_{\mathrm{T}_{n+1}^{\text {trial }}}^{\text {tra }}
$$

where $\boldsymbol{p}_{\mathbf{T}_{n+1}}^{\text {trial }}=\boldsymbol{t}_{\mathbf{T}_{n+1}}^{\mathbf{b}^{\text {trial }}} /\left\|\boldsymbol{t}_{\mathrm{T}_{n+1}}^{\mathbf{b}^{\text {trial }}}\right\|$.

Step 6. Pull-back to reference configuration and store in database. Finally, the current (one-form) frictional traction on reference configuration is obtained as the pull-back of the computed (one-form) frictional traction, defined as

$$
\boldsymbol{t}_{\mathrm{T}_{n+1}}^{\mathrm{b}^{\mathrm{ref}}}:=\boldsymbol{\varphi}^{(2) *}\left(\boldsymbol{t}_{\mathrm{T}_{n+1}}^{\mathrm{b}}\right)=\left(\boldsymbol{\tau}_{n+1}^{\alpha^{\mathrm{Ief}}} \otimes \boldsymbol{\tau}_{\alpha_{n+1}}\right) \cdot \boldsymbol{t}_{\mathrm{T}_{n+1}}^{\mathrm{b}}, \quad \alpha=1,2
$$

The current frictional traction on reference configuration is stored in the database to be used, after convergence was achieved, as initial condition for the next step.

A summary of the frictional time integration at finite deformations is shown in Table A.1.

\section{Appendix B. Linearization of the algorithm}

The frictional time integration algorithm described in Appendix A is amenable to exact linearization, allowing to compute the consistent or algorithmic tangent operator.

Linearization is performed throughout a systematic and straightforward use of the directional derivative. As the computations are quite involved, here we will provide only the key expressions and we will focus on the linearization of the trial frictional traction, which is the main point of the new algorithm.

The linearization of the new time-stepping algorithm is quite involved and requires a systematic use of the directional derivative. Here, we will focus on the linearization of the trial frictional traction one-form components $t_{\mathrm{T}_{\alpha_{n+1}}}^{\text {tria }}$ leading to the non-symmetric operator components $\Xi_{\alpha \beta}$. 
Taking the directional derivative of (A.31)-(A.32) the linearization of the new time-stepping algorithm described above takes the form:

$$
\Delta t_{\mathrm{T}_{n+1}}=\left\{\begin{array}{ll}
\Delta t_{\mathrm{T}_{n+1}}^{\text {trial }} & \text { (stick) } \\
\mu \Delta t_{\mathrm{N}_{n+1}} & p_{\mathrm{T}_{n+1_{\alpha}}}^{\text {trial }}+\mu t_{\mathrm{N}_{n+1}} \Delta p_{\mathrm{T}_{n+1}}^{\text {trial }}
\end{array}\right. \text { (slip) }
$$

with

$$
\begin{aligned}
& \Delta t_{\mathrm{N}_{n+1}}:=\epsilon_{\mathrm{N}} H\left(g_{\mathrm{N}_{n+1}}\right) \Delta g_{\mathrm{N}_{n+1}} \\
& \Delta g_{\mathrm{N}_{n+1}}:=-\left[\Delta \varphi^{(1) h}-\Delta \varphi^{(2) h} \circ \boldsymbol{\psi}_{0}^{(2)}\left(\bar{\xi}_{n+1}\right)\right] \cdot \boldsymbol{\nu} \\
& \Delta t_{\mathrm{T}_{n+1}}^{\text {trial }}:=\Xi_{\alpha \beta} \Delta \bar{\xi}_{n+1}^{\beta} \\
& \Delta p_{\mathrm{T}_{n+1}}^{\text {trial }}:=\left(\delta_{\alpha}^{\beta}-\pi_{\alpha}^{\beta}\right) \frac{\Delta t_{\mathrm{T}_{n+1_{\beta}}}^{\text {trial }}}{\left\|t_{\mathrm{T}_{n+1}}^{\text {trial }}\right\|}+\pi_{\alpha}^{\beta} p_{\mathrm{T}_{n+1}}^{b^{\text {trial }}} \cdot\left[\Delta \varphi_{, \beta}^{(2) h}(\bar{\xi})+\boldsymbol{e}_{\beta, \gamma}(\bar{\xi}) \Delta \bar{\xi}_{n+1}^{\gamma}\right] \\
& \Delta \bar{\xi}_{n+1}^{\alpha}:=A^{\alpha \beta}\left\{\left[\Delta \varphi^{(1) h}-\Delta \varphi^{(2) h}\left(\bar{\xi}_{n+1}\right)\right] \cdot \tau_{\beta}-g_{\mathrm{N}_{n+1}} \nu \cdot\left[\Delta \varphi_{, \beta}^{(2) h}\left(\bar{\xi}_{n+1}\right)\right]\right\}
\end{aligned}
$$

where $H(\cdot)$ is the Heaviside function, $\pi_{\alpha}^{\beta}$ is defined as

$$
\pi_{\alpha}^{\beta}=p_{\mathrm{T}_{n+1}}^{\text {trial }} p_{\mathrm{T}_{n+1}}^{\text {trial } \beta}
$$

and $A^{\alpha \beta}$ are the components of the inverse matrix $A^{-1}=\left[A^{\alpha \beta}\right]$, where

$$
A_{\alpha \beta}=m_{\alpha \beta}+g_{\mathrm{N}} \kappa_{\alpha \beta}
$$

Here, the non-symmetric operator $\Xi_{\alpha \beta}$ arises from the linearization of the trial frictional traction given by (A.29) and takes the form:

$$
\Xi_{\alpha \beta}:=\bar{\Xi}_{\alpha \beta}+\epsilon_{\mathrm{T}} \Gamma_{, \beta}^{\mathrm{slip}} \hat{\tau}_{1_{n+1}}^{\mathrm{ref}} \cdot \boldsymbol{\tau}_{\alpha_{n+1}}^{\mathrm{ref}}
$$

where the non-symmetric operator $\bar{\Xi}_{\alpha \beta}$ arises from the linearization of the parallel transport of $\boldsymbol{t}_{\mathrm{T}_{n}}^{\text {ref }}$ from $\overline{\boldsymbol{\xi}}_{n}$ to $\overline{\boldsymbol{\xi}}_{n+1}$ and takes the form:

$$
\begin{aligned}
\bar{\Xi}_{\alpha \beta}:= & \left(\boldsymbol{t}_{\mathrm{T}_{n}}^{\mathrm{ref}} \cdot \hat{\boldsymbol{\tau}}_{\gamma_{n}, \beta}^{\mathrm{ref}}\right)\left(\hat{\boldsymbol{\tau}}_{\gamma_{n+1}}^{\mathrm{ref}} \cdot \boldsymbol{\tau}_{\boldsymbol{\alpha}_{n+1}}^{\mathrm{ref}}\right)+\left(\boldsymbol{t}_{\mathrm{T}_{n}}^{\mathrm{ref}} \cdot \hat{\boldsymbol{\tau}}_{\gamma_{n}}^{\mathrm{ref}}\right)\left[\hat{\boldsymbol{\tau}}_{\gamma_{n+1}, \beta}^{\mathrm{ref}} \cdot \boldsymbol{\tau}_{\alpha_{n+1}}^{\mathrm{ref}}+\hat{\boldsymbol{\tau}}_{\gamma_{n+1}}^{\mathrm{ref}} \cdot \boldsymbol{E}_{\alpha, \beta}\left(\overline{\boldsymbol{\xi}}_{n+1}\right)\right] \\
& +\boldsymbol{\epsilon}_{\mathrm{T}} \Gamma^{\mathrm{slip}}\left[\hat{\boldsymbol{\tau}}_{1_{n+1}, \beta}^{\mathrm{ref}} \cdot \boldsymbol{\tau}_{\alpha_{n+1}}^{\mathrm{ref}}+\hat{\boldsymbol{\tau}}_{1_{n+1}}^{\mathrm{ref}} \cdot \boldsymbol{E}_{\boldsymbol{\alpha}, \boldsymbol{\beta}}\left(\overline{\boldsymbol{\xi}}_{n+1}\right)\right]
\end{aligned}
$$

The computation of the term $\Gamma_{, \beta}^{\text {slip }}$, associated to the variation of the slip amount, is quite involved and a detailed description is included next.

(A) Basic geometric objects and slip kinematics. Let $\bar{Y}_{n} \in \Gamma^{(2)}$ and $\overline{\boldsymbol{Y}}_{n+1} \in \Gamma^{(2)}$ be the reference placement of the closest-point projection of a typical slave particle $X \in \Gamma^{(1)}$ onto the placements $\gamma_{n}^{(2)}:=\varphi_{n}^{(2)}\left(\Gamma^{(2)}\right)$ and $\gamma_{n+1}^{(2)}:=\varphi_{n+1}^{(2)}\left(\Gamma^{(2)}\right)$ of the master surface $\Gamma^{(2)}$ at times $t_{n}$ and $t_{n+1}$, respectively.

Consider a local contact surface parametrization such that $\overline{\boldsymbol{Y}}_{n}:=\boldsymbol{\psi}_{0}^{e_{n}(2)}\left(\overline{\boldsymbol{\xi}}_{n}\right)$ and $\overline{\boldsymbol{Y}}_{n+1}:=$ $\boldsymbol{\psi}_{0}^{e_{n+1}(2)}\left(\overline{\boldsymbol{\xi}}_{n+1}\right)$, where, in general, $\boldsymbol{\psi}_{0}^{e_{n}(2)}$ and $\boldsymbol{\psi}_{0}^{e_{n+1}(2)}$ will not coincide, i.e. the reference placements $\overline{\boldsymbol{Y}}_{n}$ and $\bar{Y}_{n+1}$ will not lie, in general, on the same surface finite element and thus will be described using different local parametrization maps.

With the above notation in hand consider the Euclidean distance vector in reference configuration $d^{\text {ref }}$, with Euclidean norm denoted as $d^{\text {ref }}$ and unit vector $\boldsymbol{m}^{\text {ref }}$, defined as

$$
\boldsymbol{d}^{\mathrm{ref}}:=\overline{\boldsymbol{Y}}_{n+1}-\overline{\boldsymbol{Y}}_{n}, \quad d^{\mathrm{ref}}:=\left\|d^{\mathrm{ref}}\right\|, \quad m^{\mathrm{ref}}:=d^{\mathrm{ref}} / d^{\mathrm{ref}}
$$

Following the steps described in Section 2 , we construct two sets of local orthonormal frames attached each one to the material points $\overline{\boldsymbol{Y}}_{n}$ and $\overline{\boldsymbol{Y}}_{n+1}$, respectively, as follows: 


$$
\begin{aligned}
& \hat{\boldsymbol{\tau}}_{2_{n}}:=\frac{\boldsymbol{\nu}_{n}^{\text {ref }} \times \boldsymbol{m}^{\text {ref }}}{\left\|\boldsymbol{\nu}_{n}^{\text {ref }} \times \boldsymbol{m}^{\text {ref }}\right\|}, \quad \hat{\boldsymbol{\tau}}_{1_{n}}:=\hat{\boldsymbol{\tau}}_{2_{n}} \times \boldsymbol{\nu}_{n}^{\text {ref }} \\
& \hat{\boldsymbol{\tau}}_{2_{n+1}}:=\frac{\boldsymbol{\nu}_{n+1}^{\text {ref }} \times \boldsymbol{m}^{\text {ref }}}{\left\|\boldsymbol{\nu}_{n+1}^{\text {ref }} \times \boldsymbol{m}^{\text {ref }}\right\|}, \quad \hat{\boldsymbol{\tau}}_{1_{n+1}}:=\hat{\boldsymbol{\tau}}_{2_{n+1}} \times \boldsymbol{\nu}_{n+1}^{\text {ref }}
\end{aligned}
$$

Here, $\boldsymbol{\nu}_{n}^{\text {ref }}$ and $\boldsymbol{\nu}_{n+1}^{\text {ref }}$ are the unit normal vectors to the reference placement of the master surface at the points $\overrightarrow{\boldsymbol{Y}}_{n}$ and $\overline{\boldsymbol{Y}}_{n+1}$, respectively. Using the convective basis $\left\{\boldsymbol{\tau}_{1_{n}}^{\text {ref }}, \boldsymbol{\tau}_{2_{n}}^{\text {ref }}\right\}$ and $\left\{\boldsymbol{\tau}_{1_{n+1}}^{\text {ref }}, \boldsymbol{\tau}_{2_{n+1}}^{\text {ref }}\right\}$ induced by the tangent maps $D \boldsymbol{\psi}_{0}^{e_{n}(2)}$ and $D \boldsymbol{\psi}_{0}^{e_{n+1}(2)}$ the unit normals at the points $\overline{\boldsymbol{Y}}_{n}$ and $\overline{\boldsymbol{Y}}_{n+1}$ take the form

$$
\begin{aligned}
& \boldsymbol{\nu}_{n}^{\text {ref }}:=\left(\boldsymbol{\tau}_{1_{n}}^{\text {ref }} \times \boldsymbol{\tau}_{2_{n}}^{\text {ref }}\right) /\left\|\boldsymbol{\tau}_{1_{n}}^{\text {ref }} \times \boldsymbol{\tau}_{2_{n}}^{\text {ref }}\right\| \\
& \nu_{n+1}^{\text {ref }}:=\left(\boldsymbol{\tau}_{1_{n+1}}^{\text {ref }} \times \boldsymbol{\tau}_{2_{n+1}}^{\text {ref }}\right) /\left\|\boldsymbol{\tau}_{1_{n+1}}^{\text {ref }} \times \boldsymbol{\tau}_{2_{n+1}}^{\text {ref }}\right\|
\end{aligned}
$$

(B) Assumed slip path and slip length computation. Using the local orthonormal frames defined above, one constructs an assumed slip path on the basis of the unit tangent vectors $\hat{\tau}_{1_{n}}$ and $\hat{\tau}_{1_{n+1}}$ at the given initial and final points $\overline{\boldsymbol{Y}}_{n}$ and $\overline{\boldsymbol{Y}}_{n+1}$, respectively. The slip curve is defined through the introduction of Hermitian shape functions via the map:

$$
\zeta \in[-1,+1] \mapsto \bar{Y}(\zeta):=\mathscr{H}^{1}(\zeta) \bar{Y}_{n}+\frac{d^{\mathrm{ref}}}{2 \cos \Theta_{n}} \overline{\mathscr{H}}^{1}(\zeta) \hat{\tau}_{1_{n}}+\mathscr{H}^{2}(\zeta) \overline{\boldsymbol{Y}}_{n+1}+\frac{d^{\mathrm{ref}}}{2 \cos \Theta_{n+1}} \overline{\mathscr{H}}^{2}(\zeta) \hat{\tau}_{1_{n+1}}
$$

where $\cos \Theta_{n}:=\hat{\tau}_{1_{n}} \cdot \boldsymbol{m}^{\text {ref }}$ and $\cos \Theta_{n+1}:=\hat{\tau}_{1_{n+1}} \cdot \boldsymbol{m}^{\text {ref }}$ and the Hermite shape functions are given in (2.3).

The slip curve defined by (B.10) can be viewed as an approximation to the geodesic curve through the points $\bar{Y}_{n}$ and $\overline{\boldsymbol{Y}}_{n+1}$ of the master surface $\Gamma^{(2) h}$.

The tangent map to the curve defined by (B.10) takes the form:

$$
\begin{aligned}
\zeta \in[-1,+1] \mapsto \overline{\boldsymbol{Y}}_{\mathrm{T}}(\zeta):= & \nabla \mathscr{H}^{1}(\zeta) \overline{\boldsymbol{Y}}_{n}+\frac{d^{\mathrm{ref}}}{2 \cos \Theta_{n}} \nabla \overline{\mathscr{H}}^{1}(\zeta) \hat{\tau}_{1_{n}} \\
& +\nabla \mathscr{H}^{2}(\zeta) \overline{\boldsymbol{Y}}_{n+1}+\frac{d^{\mathrm{ref}}}{2 \cos \Theta_{n+1}} \nabla \overline{\mathscr{H}}^{2}(\zeta) \hat{\tau}_{1_{n+1}}
\end{aligned}
$$

where $\nabla \mathscr{H}^{A}(\zeta):=\mathrm{d} \mathscr{H}^{A}(\zeta) / \mathrm{d} \zeta$ and $\nabla \overline{\mathscr{H}}^{A}(\zeta):=\mathrm{d} \overline{\mathscr{H}}^{A}(\zeta) / \mathrm{d} \zeta$ for $A=1,2$. In order to simplify the notation in further developments we will introduce the following scalar definitions

$$
\bar{Y}_{\mathrm{T}_{n}}:=\frac{d^{\mathrm{ref}}}{2 \cos \Theta_{n}}, \quad \bar{Y}_{\mathrm{T}_{n+1}}:=\frac{d^{\mathrm{ref}}}{2 \cos \Theta_{n+1}}
$$

Note that actually these definitions are such that $\bar{Y}_{\mathrm{T}_{n}}=\left\|\overline{\boldsymbol{Y}}_{\mathrm{T}}(\zeta=-1)\right\|=:\left\|\overline{\boldsymbol{Y}}_{\mathrm{T}_{n}}\right\|$ and $\bar{Y}_{\mathrm{T}_{n+1}}=\| \overline{\boldsymbol{Y}}_{\mathrm{T}}(\zeta=$ 1) $\|=:\| \overline{\boldsymbol{Y}}_{\mathrm{T}_{n+1}} \|$.

Using the tangent map defined above, the slip curve length can be expressed as

$$
\Gamma^{\text {slip }}=\int_{-1}^{1}\left\|\overline{\boldsymbol{Y}}_{\mathrm{T}}(\zeta)\right\| \mathrm{d} \zeta
$$

This integral can be numerically evaluated using a quadrature rule, leading to an expression of the form

$$
\Gamma^{\text {slip }}=\sum_{I=1}^{N_{\text {int }}} W_{I}\left\|\overline{\boldsymbol{Y}}_{\mathrm{T}}\left(\zeta_{I}\right)\right\|
$$

where $\zeta_{I} \in[-1,+1]$ are the positions of the integration points in the isoparametric domain $[-1,+1], W_{I}$ are the integration weights and $N_{\text {int }}$ is the number of integration points to be uscd in the quadraturc rule. 
(C) Linearization of the kinematics. The linearization of the frictional time integration algorithm presented in Appendix A requires the linearization of the local orthonormal frames introduced above.

(i) Linearization of the (normalized) distance vector in reference configuration. The linearization of the Euclidean distance vector $\boldsymbol{d}^{\text {ref }}$, distance magnitude $d^{\text {ref }}$ and normalized distance vector $\boldsymbol{m}^{\text {ref }}$, can be written in terms of the variation of the isoparametric coordinates $\delta \bar{\xi}^{\alpha}$ as

$$
\begin{aligned}
& \delta d^{\text {ref }}:-\boldsymbol{d},,_{\alpha}^{\text {ref }} \delta \bar{\xi}^{\alpha} \\
& \delta d^{\text {ref }}:=d,{ }_{\alpha}^{\text {ref }} \delta \bar{\xi}^{\alpha} \\
& \delta m^{\text {ref }}:=m,{ }_{\alpha}^{\text {ref }} \delta \bar{\xi}^{\alpha}
\end{aligned}
$$

where

$$
\begin{aligned}
\boldsymbol{d},{ }_{\alpha}^{\text {ref }} & :=\boldsymbol{\tau}_{\alpha_{n+1}}^{\text {ref }} \\
d_{,_{\alpha}}^{\text {ref }} & :=\boldsymbol{m}^{\text {ref }} \cdot \boldsymbol{\tau}_{\alpha_{n+1}}^{\text {ref }} \\
\boldsymbol{m}_{{ }_{\alpha}}^{\text {ref }}: & :=\frac{1}{d^{\text {ref }}} \boldsymbol{P}_{\boldsymbol{m}^{\text {ref }}}\left[\boldsymbol{\tau}_{\alpha_{n+1}}^{\text {ref }}\right]
\end{aligned}
$$

Here, $\mathbf{P}_{m^{\mathrm{ref}}}[\bullet]$ is the projection operator defined as

$$
\mathbf{P}_{m^{\text {ref }}}[\bullet]:=\left(1-m^{\mathrm{ref}} \otimes \boldsymbol{m}^{\mathrm{ref}}\right) \cdot[\bullet]
$$

(ii) Linearization of the local orthonormal frame attached to the point $\overline{\boldsymbol{Y}}_{n}$. The linearized variation of the local orthonormal frame $\left\{\hat{\boldsymbol{\tau}}_{1_{n}}, \hat{\boldsymbol{\tau}}_{2_{n}}, \boldsymbol{\nu}_{n}^{\text {ref }}\right\}$ attached to the point $\overline{\boldsymbol{Y}}_{n}$ on reference configuration, takes the form

$$
\begin{aligned}
& \delta \hat{\tau}_{1_{n}}:=\hat{\tau}_{1_{n}, \alpha} \delta \bar{\xi}^{\alpha} \\
& \delta \hat{\tau}_{2_{n}}:=\hat{\tau}_{2_{n}, \alpha} \delta \bar{\xi}^{\alpha} \\
& \delta \nu_{n}^{\text {ref }}:=\nu_{n, \alpha}^{\text {ref }} \delta \bar{\xi}^{\alpha}
\end{aligned}
$$

where

$$
\begin{aligned}
& \hat{\boldsymbol{\tau}}_{1_{n}, \alpha}:=\hat{\boldsymbol{\tau}}_{2_{n}, \alpha} \times \boldsymbol{\nu}_{n}^{\text {ref }} \\
& \hat{\boldsymbol{\tau}}_{2_{n}, \alpha}:=\frac{1}{\| \boldsymbol{\nu}_{n}^{\text {ref }} \times \boldsymbol{m}^{\text {ref } \|}} \mathbf{P}_{\hat{\tau}_{2_{n}}}\left[\boldsymbol{\nu}_{n}^{\text {ref }} \times \boldsymbol{m}_{, \alpha}^{\text {ref }}\right] \\
& \boldsymbol{\nu}_{n, \alpha}^{\text {ref }}:=0
\end{aligned}
$$

Here, the projection operator $\mathbb{P}_{\hat{\tau}_{2_{n}}}[\bullet]$ is defined as

$$
\mathbf{P}_{\hat{\tau}_{2_{n}}}[\bullet]:=\left(1-\hat{\tau}_{2_{n}} \otimes \hat{\tau}_{2_{n}}\right) \cdot[\bullet]
$$

(iii) Linearization of the local orthonormal frame attached to the point $\bar{Y}_{n+1}$. The linearized variation of the local orthonormal frame $\left\{\hat{\tau}_{1_{n+1}}, \hat{\tau}_{2_{n+1}}, \boldsymbol{\nu}_{n+1}^{\text {ref }}\right\}$ attached to the point $\boldsymbol{Y}_{n+1}$ on reference configuration, takes the form

$$
\begin{aligned}
& \delta \hat{\tau}_{1_{n+1}}:=\hat{\tau}_{1_{n+1}, \alpha} \delta \bar{\xi}^{\alpha} \\
& \delta \hat{\tau}_{2_{n+1}}:=\hat{\tau}_{2_{n+1}, \alpha} \delta \bar{\xi}^{\alpha} \\
& \delta \nu_{n+1}^{\text {ref }}:=\nu_{n+1, \alpha}^{\text {ref }} \delta \bar{\xi}^{\alpha}
\end{aligned}
$$

where 


$$
\begin{aligned}
& \hat{\tau}_{1_{n+1}, \alpha}:=\hat{\tau}_{2_{n+1}, \alpha} \times \nu_{n+1}^{\text {ref }}+\hat{\tau}_{2_{n+1}} \times \nu_{n+1, \alpha}^{\text {ref }} \\
& \hat{\boldsymbol{\tau}}_{2_{n+1}, \alpha}:=\frac{1}{\left\|\boldsymbol{\nu}_{n+1}^{\text {ref }} \times \boldsymbol{m}^{\text {ref }}\right\|} \mathbf{P}_{\hat{\tau}_{2_{n+1}}}\left[\boldsymbol{\nu}_{n+1, \alpha}^{\text {ref }} \times \boldsymbol{m}^{\text {ref }}+\boldsymbol{\nu}_{n+1}^{\text {ref }} \times \boldsymbol{m}_{\alpha}^{\text {ref }}\right] \\
& \boldsymbol{\nu}_{n+1, \alpha}^{\text {ref }}:=\frac{1}{\left\|\boldsymbol{\tau}_{1_{n+1}}^{\text {ref }} \times \boldsymbol{\tau}_{2_{n+1}}^{\text {ref }}\right\|} \mathbb{P}_{\nu_{n+1}^{\text {ref }}}\left[E_{1, \alpha}\left(\bar{\xi}_{n+1}\right) \times \tau_{2_{n+1}}^{\text {ref }}+\boldsymbol{\tau}_{l_{n+1}^{\text {ref }}}^{\text {ref }} \times E_{2, \alpha}\left(\bar{\xi}_{n+1}\right)\right]
\end{aligned}
$$

Here, the projection operators $\mathbb{P}_{\tau_{2_{n 1}}}[\bullet]$ and $\mathbb{P}_{\nu_{n+1}^{\text {ref }}}[\bullet]$ are defined as

$$
\begin{aligned}
& \mathbb{P}_{\hat{\tau}_{2_{n}}}[\bullet]:=\left(\mathbf{1}-\hat{\tau}_{2_{n}} \otimes \hat{\tau}_{2_{n}}\right) \cdot[\bullet] \\
& \mathbf{P}_{\nu_{n+1}^{\text {ref }}}[\bullet]:=\left(\mathbf{1}-\nu_{n+1}^{\text {ref }} \otimes \nu_{n+1}^{\text {ref }}\right) \cdot[\bullet]
\end{aligned}
$$

(iv) Linearization of the slip length. The linearized variation of the slip curve length given by (B.14) takes the form:

$$
\delta \Gamma^{\text {slip }}:=\Gamma_{, \alpha}^{\text {slip }} \delta \bar{\xi}^{\alpha}
$$

where

$$
\Gamma_{. \alpha}^{\text {slip }}:=\sum_{I-1}^{N_{\text {int }}} W_{l} \frac{\overline{\boldsymbol{Y}}_{\mathrm{T}}\left(\zeta_{l}\right)}{\left\|\overline{\boldsymbol{Y}}_{\mathrm{T}}\left(\zeta_{l}\right)\right\|} \cdot \overline{\boldsymbol{Y}}_{\mathrm{T}, \boldsymbol{\alpha}}\left(\zeta_{l}\right)
$$

Here, $\overline{\boldsymbol{Y}}_{\mathrm{T}, \alpha}\left(\zeta_{I}\right)$ can be computed from (B.11) and takes the form:

$$
\begin{aligned}
\overline{\boldsymbol{Y}}_{\mathrm{T}, \alpha}\left(\zeta_{I}\right):= & \nabla \mathscr{H}^{2}\left(\zeta_{I}\right) \tau_{\alpha_{n+1}}^{\text {ref }}+\nabla \overline{\mathscr{H}}^{1}\left(\zeta_{I}\right)\left[\bar{Y}_{\mathrm{T}_{n}, \alpha} \hat{\tau}_{1_{n}}+\bar{Y}_{\mathrm{T}_{n}} \hat{\tau}_{\mathrm{I}_{n, \alpha}}\right] \\
& +\nabla \overline{\mathscr{K}}^{2}\left(\zeta_{I}\right)\left[\bar{Y}_{\mathrm{T}_{n+1}, \alpha} \hat{\tau}_{1_{n+1}}+\bar{Y}_{\mathrm{T}_{n+1}} \hat{\tau}_{1_{n+1 . \alpha}}\right]
\end{aligned}
$$

where

$$
\begin{aligned}
& \bar{Y}_{\mathrm{T}_{n, \alpha}}:=\frac{d_{, \alpha}^{\mathrm{ref}}}{2 \cos \Theta_{n}}-\frac{d^{\mathrm{ref}}}{2 \cos ^{2} \Theta_{n}}\left[\cos \Theta_{n}\right]_{, \alpha} \\
& \bar{Y}_{\mathrm{T}_{n+1, \alpha}}:=\frac{d_{, \alpha}^{\mathrm{ref}}}{2 \cos \Theta_{n+1}}-\frac{d^{\mathrm{ref}}}{2 \cos ^{2} \Theta_{n+1}}\left[\cos \Theta_{n+1}\right]_{, \alpha}
\end{aligned}
$$

and

$$
\begin{aligned}
& {\left[\cos \Theta_{n}\right]_{, \alpha}:=\boldsymbol{m}_{, \alpha}^{\text {ref }} \cdot \hat{\boldsymbol{\tau}}_{1_{n}}+\boldsymbol{m}^{\text {ref }} \cdot \hat{\boldsymbol{\tau}}_{1_{n, \alpha}}} \\
& {\left[\cos \Theta_{n+1}\right]_{, \alpha}:=\boldsymbol{m}_{, \alpha}^{\mathrm{ref}} \cdot \hat{\boldsymbol{\tau}}_{1_{n+1}}+\boldsymbol{m}^{\mathrm{ref}} \cdot \hat{\boldsymbol{\tau}}_{1_{n+1, \alpha}}}
\end{aligned}
$$

\section{References}

[1] G. Duvaut and J.L. Lions, Les Inequations en Mecanique et en Physique (Dunod, Paris, 1972).

[2] N. Kikuchi and J.T. Oden, Contact Problems in Elasticity: A Study of Variational Inequalities and Finite Element Methods (SIAM, Philadelphia, 1988).

[3] J.T. Oden and E.B. Pires, Algorithms and numerical results for finite element approximations of contact problems with non-classical friction laws, Comput. Struct. 19 (1984) 137-147.

[4] J.-H. Cheng and N. Kikuchi, An analysis of metal forming processes using large deformation elastic-plastic formulations, Comput. Methods Appl. Mech. Engrg. 49 (1985) 71-108.

[5] J.O. Hallquist, G.L. Goudreau and D.J. Benson, Sliding interfaces with contact-impact in large-scale Lagrangian computations, Comput. Methods Appl. Mech. Engrg. 51 (1985) 107-137.

[6] J.C. Simo, P. Wriggers and R.L. Taylor, A perturbed Lagrangian formulation for the finite element solution of contact problems. Comput. Methods Appl. Mech. Engrg. 50 (1985) 163-180.

[7] A. Curnier and P. Alart, A generalized Newton method for contact problems with friction, J. Mecanique Theorique Appl. suppl. no. 1 to 7 (1988) 67-82. 
[8] P. Wriggers, T. Vu Van and E. Stein, Finite element formulation of large deformation impact-contact problems with friction, Comput. Struct. 37 (1990) 319-331.

[9] T. Belytschko and M.O. Neal, Contact-impact by the pinball algorithm with penalty and Lagrangian methods, Int. J. Numer. Methods Engrg. Methods Engrg. (1991) to appear.

[10] T.A. Laursen, Formulation and treatment of frictional contact problems using finite elements, Ph.D. Dissertation, Stanford University, Division of Applied Mechanics, Report no. 92-6, 1992.

[11] T.A. Laursen and J.C. Simo, Formulation and regularization of frictional contact problems for Lagrangian finite element computations, in: D.R.J. Owen, E. Onate and E. Hinton, eds,, Proc. Third Int. Conf. on Computational Plasticity: Fundamentals and Applications, COMPLAS III (Pineridge Press, Swansea, 1992) 395-407.

[12] T.A. Laursen and J.C. Simo, A continuum-based finite element formulation for the implicit solution of multi-body, large deformation frictional contact problems, Int. J. Numer. Methods Engrg. 36 (1993) 3451-3485.

[13] C. Agelet de Saracibar, Numerical analysis of frictional wear contact problems: Computational aspects and applications (1995), to appear.

[14] C. Agelet de Saracibar, Numerical analysis of frictional contact problems, Lecture Notes of Short Course on Computational Techniques for Plasticity, International Center for Numerical Methods in Engineering, Barcelona, Spain, 1995.

[15] J.C. Simo and T.A. Laursen, An augmented Lagrangian treatment of contact problems involving friction, Comput. Struct. 42 (1992) $97-116$.

[16] T.A. Laursen and J.C. Simo, Algorithmic symmetrization of coulomb frictional problems using augmented Lagrangians, Comput. Methods Appl. Mech. Engrg. (1994), to appear.

[17] T.A. Laursen and S. Govindjee, A note on the treatment of frictionless contact between non-smooth surfaces in fully non-linear problems, Comm. Appl. Numer. Methods 10 (1994) 869-878.

[18] P. Wriggers and J.C. Simo, A note on tangent stiffness for fully nonlinear contact problems, Comm. Appl. Methods 1 (1985) 199-203.

[19] H. Parisch, A consistent tangent stiffness matrix for three-dimensional non-linear contact analysis, Int. J. Numer. Methods Engrg. 28 (1989) 1803-1812.

[20] P. Wriggers, On consistent tangent matrices for frictional contact problems, in: J. Middleton et al., eds., Proceedings of the International Conference NUMETA '87 (A.A. Balkema, Rotterdam, 1987).

[21] J.C. Simo and T.J.R. Hughes, Elastoplasticity and Viscoplasticity: Computational Aspects (Springer-Verlag, Berlin, 1994) to be published.

[22] J.C. Simo, Numerical analysis aspects of plasticity, in: P.G. Ciarlet and J.J. Lions, eds., Handbook of Numerical Analysis, Vol. IV (North-Holland, 1994).

[23] A.E. Giannakopoulos, The return mapping method for the integration of friction constitutive relations, Comput. Struct. 32 (1989) 157-167.

[24] O.C. Zienkiewicz and R.L. Taylor, The Finite Element Method, 4th edition, Vol. 2: Solid and Fluid Mechanics, Dynamics and Non-linearity (McGraw-Hill, London, 1991). 